\title{
The Riemann-Hilbert Problem for Holonomic Systems
}

\author{
By
}

\author{
Masaki KASHIWARA*
}

\section{Introduction}

The purpose of this paper is to give a proof to the equivalence of the derived category of holonomic systems and that of constructible sheaves.

Let $X$ be a paracompact complex manifold and let $\mathscr{D}_{X}$ and $\mathcal{O}_{X}$ be the sheaf of differential operators and holomorphic functions, respectively.

We denote by $\operatorname{Mod}\left(\mathscr{D}_{X}\right)$ the abelian category of left $\mathscr{D}_{X}$-Modules and by $\mathrm{D}\left(\mathscr{D}_{X}\right)$ its derived category. Let $\mathrm{D}_{\mathrm{rh}}^{\mathrm{b}}\left(\mathscr{D}_{X}\right)$ denote the full sub-category of $\mathrm{D}\left(\mathscr{D}_{X}\right)$ consisting of bounded complexes whose cohomology groups are regular holonomic $([\mathrm{KK}])$.

By replacing $\mathscr{D}_{X}$ with $\mathscr{D}_{X}^{\infty}$, the sheaf of differential operators of infinite order, and "regular holonomic" with "holonomic," we similarly define $\operatorname{Mod}\left(\mathscr{D}_{X}^{\infty}\right), \mathrm{D}\left(\mathscr{D}_{X}^{\infty}\right)$ and $\mathrm{D}_{\mathrm{h}}^{\mathrm{b}}\left(\mathscr{D}_{X}^{\infty}\right)$.

Let us denote by $\operatorname{Mod}(X)$ the category of sheaves of $\boldsymbol{C}$-vector spaces on $X$ and by $\mathrm{D}(X)$ its derived category. We denote by $\mathrm{D}_{\mathrm{c}}^{\mathrm{b}}(X)$ the full sub-category of $\mathrm{D}(X)$ consisting of bounded complexes whose cohomology groups are constructible.

Let us define

$$
\begin{aligned}
& J_{X}: \mathrm{D}_{\mathrm{rh}}^{\mathrm{b}}\left(\mathscr{D}_{X}\right) \rightarrow \mathrm{D}_{\mathrm{h}}^{\mathrm{b}}\left(\mathscr{D}_{X}^{\infty}\right) \\
& D R_{X}^{\infty}: \mathrm{D}_{\mathrm{h}}^{\mathrm{b}}\left(\mathscr{D}_{X}\right) \rightarrow \mathrm{D}_{\mathrm{c}}^{\mathrm{b}}(X) \\
& D R_{X}=D R_{X}^{\infty} J_{X}: D_{\mathrm{rh}}^{\mathrm{b}}\left(\mathscr{D}_{X}\right) \rightarrow \mathrm{D}_{\mathrm{c}}^{\mathrm{b}}(X)
\end{aligned}
$$

by

$$
\begin{aligned}
& J_{X}=\mathscr{D}_{X}^{\infty} \otimes \\
& D R_{X}^{\infty}: \mathscr{M} \mapsto \boldsymbol{R} \mathscr{H}_{X} m_{\mathscr{D}_{X}^{\infty}}\left(\mathcal{O}_{X}, \mathscr{M}\right)
\end{aligned}
$$

Received March 4, 1983.

* Research Institute for Mathematical Sciences, Kyoto University, Kyoto 606, Japan. 


$$
D R_{X}: \mathscr{M} \mapsto \boldsymbol{R} \mathscr{H}_{0} m_{\mathscr{D}_{X}}\left(\mathcal{O}_{X}, \mathscr{M}\right) .
$$

The purpose of this paper is to prove the following theorem.

Theorem. By $J_{X}, D R_{X}, D R_{X}^{\infty}, \mathrm{D}_{\mathrm{rh}}^{\mathrm{b}}\left(\mathscr{D}_{X}\right), \mathrm{D}_{\mathrm{h}}^{\mathrm{b}}\left(\mathscr{D}_{X}^{\infty}\right)$ and $\mathrm{D}_{\mathrm{c}}^{\mathrm{b}}(X)$ are equivalent.

This result was announced in $[\mathrm{K}]$. Mebkout [Me] gave another proof to this theorem.

0. 2. In $[\mathrm{KK}]$, we have already shown that $J_{X}$ gives an equivalence and that $D R_{X}$ is fully faithful; i. e. for any $\mathscr{M}^{\circ}, \mathscr{M}^{\prime} \in \mathrm{D}_{\mathrm{rh}}^{\mathrm{b}}\left(\mathscr{D}_{X}\right)$, we have

$$
\begin{aligned}
& \operatorname{Hom}_{D_{\mathrm{rh}}^{\mathrm{b}}\left(\mathscr{D}_{X}\right)}\left(\mathscr{M}^{\prime}, \mathscr{M}^{* \prime}\right) \\
\widetilde{H^{\prime}} & \operatorname{Hom}_{\mathrm{D}_{\mathrm{c}}^{\mathrm{b}}(X)}\left(D R_{X}\left(\mathscr{M}^{*}\right), D R_{X}\left(\mathscr{M}^{* \prime}\right)\right) .
\end{aligned}
$$

0. 3. We define $*: \mathrm{D}_{\mathrm{rh}}^{\mathrm{b}}\left(\mathscr{D}_{X}\right) \rightarrow \mathrm{D}_{\mathrm{rh}}^{\mathrm{b}}\left(\mathscr{D}_{X}\right)^{\circ}$ by $\mathscr{M}^{\bullet} \mapsto \boldsymbol{R} \mathscr{H} o m_{\mathscr{D}_{X}}\left(\mathscr{M}^{\circ}, \mathscr{D}_{X}\right)$ $\bigotimes_{\mathscr{D}_{X}}^{\otimes-1}[\operatorname{dim} X]$, and $*: \mathrm{D}_{\mathrm{c}}^{\mathrm{b}}(X) \rightarrow \mathrm{D}_{\mathrm{c}}^{\mathrm{b}}(X)^{\circ}$ by $F^{*} \mapsto \boldsymbol{R} \mathscr{H}_{0} m_{c_{X}}\left(F^{*}, \boldsymbol{C}_{X}\right)$. Here $\circ$ denotes the opposite category and $\Omega_{X}$ denotes the sheaf of differential forms with the highest degree. Then we have $D R_{X^{\circ}} *=$ $* \circ D R_{X}$ and $* *=\mathrm{id}$.

0. 4. Now for $\mathscr{M}^{\cdot} \in \mathrm{D}_{\mathrm{rh}}^{\mathrm{b}}\left(\mathscr{D}_{X}\right)$, we put $F^{\cdot}=D R_{X}\left(\mathscr{M}^{*}\right)^{*}$. Then in [KK] we have proved

$$
J_{X} \mathscr{M}^{\cdot}=\boldsymbol{R} \mathscr{H} o m_{c_{X}}\left(F^{*}, \mathcal{O}_{X}\right) .
$$

By taking the flabby resolution of $\mathcal{O}_{X}$

$$
0 \rightarrow \mathcal{O}_{X} \stackrel{\bar{\partial}}{\longrightarrow} \mathscr{B}_{X}^{(0,1)} \stackrel{\bar{\partial}}{\longrightarrow} \cdots \rightarrow \mathscr{B}_{X}^{(0, n)} \rightarrow 0
$$

of $\mathcal{O}_{X}$, we have therefore

$$
J_{X} \mathscr{M}^{\cdot}=\mathscr{H}_{0} m_{C_{X}}\left(F^{*}, \mathscr{B}_{X}^{(0, \bullet)}\right) .
$$

Here $\mathscr{B}$ denotes the sheaf of hyperfunctions.

0. 5. Keeping this in mind, we shall construct the inverse functor $\Psi_{X}: \mathrm{D}_{\mathrm{c}}^{\mathrm{b}}(X) \rightarrow \mathrm{D}_{\mathrm{rh}}^{\mathrm{b}}\left(\mathscr{D}_{X}\right)$ as follows. The idea is to replace $\mathscr{B}_{X}$ in (0.4.1) with $\mathscr{D} b_{X}$, the sheaf of distributions. However, since $F \mapsto$ $\mathscr{H} o m\left(F, \mathscr{D} b_{X}\right)$ is not an exact functor, $\mathscr{H} o m\left(F^{\cdot}, \mathscr{D} b_{X}^{(0 . \cdot)}\right)$ is not welldefined. Therefore, we have to modify $\mathscr{H} o m(*, \mathscr{D} \ell)$.

0.6. Let $M$ be a real analytic manifold and $\mathscr{D} b_{M}$ the sheaf of distributions on $M$. A sheaf $F$ of $\boldsymbol{C}$-vector spaces on $M$ is called 
$\boldsymbol{R}$-constructible, if there exists a sub-analytic (see [H1], [H2]) stratification $M=\cup M_{\alpha}$ such that $\left.F\right|_{M_{\alpha}}$ is locally constant.

We define, for an $\boldsymbol{R}$-constructible sheaf $F$, the sub-sheaf $T H(F)$ of $\mathscr{H o m}_{c_{M}}\left(F, \mathscr{D} b_{M}\right)$ as follows. For any open subset $\Omega$ of $M$ and $\varphi \in \Gamma\left(\Omega, \mathscr{H o m}_{C}\left(F, \mathscr{D} b_{M}\right)\right), \varphi$ belongs to $\Gamma(\Omega, T H(F))$ if, for any relatively compact open sub-analytic subset $U$ of $\Omega$ and $s \in \Gamma(U, F)$ there exists $u \in \Gamma\left(M, \mathscr{D} b_{M}\right)$ such that $\left.u\right|_{U}=\varphi(s)$.

Then it turns out that $F \mapsto T H(F)$ is an exact functor in $F$.

0.7. Let $X$ be a complex manifold. We shall define $\Psi_{X}: \mathrm{D}_{\mathrm{c}}^{\mathrm{b}}(X)^{\circ}$ $\rightarrow \mathrm{D}_{\mathrm{rh}}^{\mathrm{b}}\left(\mathscr{D}_{X}\right)$, which will be an inverse of $D R_{X^{\circ *}}$, as follows. For $F^{*} \in$ $\mathrm{D}_{\mathrm{c}}^{\mathrm{b}}(X)$, we shall take a bounded complex $G^{\circ}$, quasi-isomorphic to $F^{*}$, such that $G^{j}$ is $\boldsymbol{R}$-constructible for any $j$. We define $\Psi_{X}\left(F^{*}\right)$ as the Dolbeaut complex with $T H(G)$ as coefficients

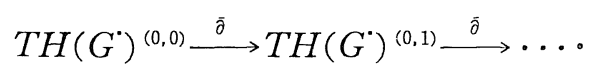

Since $T H(*)$ is an exact functor, $\Psi_{X}$ is well-defined. Once we introduced $\Psi_{X}$, it is not difficult to show that $\Psi_{X}$ and $D R_{X} \circ *$ are an inverse to each other. The main idea is to reduce the problem to a simple case by using Hironaka's desingularization theorem.

0. 8. The plan of this article is as follows. $\S 1$ is the preparation to $\S 2$, where we shall study the properties of $\boldsymbol{R}$-constructible sheaves. In $\S 3$ we define the functor $T H$. Its properties are studied in $\S 4$. In $\S 5$, we review the theory of regular holonomic systems. In $\S 6$ we announce the statement of our main theorem and $\S 7$ is devoted to its proof. In $\S 8$ we shall give some applications.

\section{$\S 1$. Constructible Sheaves on a Semi-Simplicial Complex}

1. 0 . In the later section, we treat constructible sheaves on a complex manifold. However, constructible sheaves are not easy objects to manipulate. To avoid this difficulty, we study $\mathbb{R}$-constructible sheaves (Def. 2.6) on its underlying real analytic manifold. This section is the preliminary to study $\boldsymbol{R}$-constructible sheaves.

1. 1. In this paper, a simplicial complex $\mathscr{S}=(S, \sqrt{ })$ means the following: $\mathscr{S}$ consists of two data, a set $S$ and a set $\Delta$ of subsets of $S$, which satisfy the following axioms: 
(1.1.1) Any $\sigma$ of $\Delta$ is a non-empty finite subset of $S$.

(1.1.2) If $\tau$ is a non-empty subset of an element $\sigma$ of $\Delta$, then $\tau$ belongs to $\Delta$.

(1. 1.3) For any $p \in S,\{p\}$ belongs to $\Delta$.

(1.1.4) (locally finitude) For any $p \in S$, $\{\sigma \in \Delta ; p \in \sigma\}$ is a finite set.

An element of $S$ is called a vertex and an element of $\Delta$ is called a simplex. Let $\mathbb{R}^{S}$ denote the set of maps from $S$ into $\boldsymbol{R}$, equipped with the product topology. For any $\sigma \in \Delta$, we define the subset $|\sigma|$ of $\boldsymbol{R}^{S}$ by

(1.1.5) $|\sigma|=\left\{x \in \boldsymbol{R}^{s} ; x(p)=0\right.$ for $p \notin \sigma, x(p)>0$ for $p \in \sigma$ and $\left.\sum_{p \in \sigma} x(p)=1\right\}$.

We denote by $|\mathscr{S}|$ the union of $|\sigma|(\sigma \in \Delta)$ endowed with the induced topology from the product topology of $\mathbb{R}^{S}$. Then $|\sigma|$ 's are disjoint to each other. For any $\sigma \in \Delta$, we set

$$
U(\sigma)=\bigcup_{\Delta \ni \tau \supset \sigma}|\tau|
$$

and for any $x \in|\mathscr{S}|$, we set

$$
U(x)=U(\sigma(x)),
$$

where $\sigma(x)$ is the unique simplex such that $|\sigma|$ contains $x$. Hence we have

(1.1.8) $U(x)=\{y \in|\mathscr{S}| ; y(p)>0$ for any $p \in S$ such that $x(p)>0\}$, and

$$
U(\sigma)=\{x \in|\mathscr{S}| ; x(p)>0 \text { for } p \in \sigma\} .
$$

Hence, $U(\sigma)$ and $U(x)$ are open subsets of $|\mathscr{S}|$. Define $S(\sigma)$ by

$$
S(\sigma)=\{p \in S ; \quad\{p\} \cup \sigma \in \Delta\} .
$$

Then $S(\sigma)$ is a finite subset of $S$ by (1.1.4), and $U(\sigma)$ is contained in $\boldsymbol{R}^{S(\sigma)}$. Hence $U(\sigma)$ is homeomorphic to a locally closed subset of $\boldsymbol{R}^{l}$ with $l=\# S(\sigma)$. As we have

$$
|\sigma|=\{x \in U(\sigma) ; x(p)=0 \text { for } p \notin \sigma\},
$$

$|\sigma|$ is a closed subset of $U(\sigma)$, and hence $|\sigma|$ is locally closed in $|\mathscr{S}|$. We can also verify

(1.1.12) $|\bar{\sigma}|=\left\{x \in \mathbb{R}^{S} ; x(p) \geqq 0\right.$ for any $p \in S, x(p)=0$ 


$$
\begin{aligned}
& \text { for any } \left.p \notin \sigma \text { and } \sum_{p \in \sigma} x(p)=1\right\} \\
& =\bigcup_{\sigma \supset \tau}|\tau| \text {. }
\end{aligned}
$$

Also, we have, for any $\sigma, \tau \in \Delta$,

$$
U(\sigma) \cap U(\tau)=\left\{\begin{array}{cl}
U(\sigma \cup \tau) & \text { if } \sigma \cup \tau \in \Delta \\
\varnothing & \text { if } \sigma \cup \tau \notin \Delta
\end{array}\right.
$$

$$
U(\sigma) \subset U(\tau) \text { if and only if } \sigma \supset \tau \text {. }
$$

The axiom (1.1.4) implies together with (1.1.13) that $\{U(\sigma)\}_{\sigma \in \Delta}$ is a locally finite open covering of $|\mathscr{S}|$. Therefore, $|\mathscr{S}|$ is a paracompact topological space.

1. 2. Let $\mathscr{S}=(S, \Delta)$ be a simplicial complex. We denote by $\operatorname{Mod}(\mathscr{S})$ the abelian category of sheaves of $\mathbb{C}$-vector spaces on $|\mathscr{S}|$.

Definition 1. 1. A sheaf $F$ of $\boldsymbol{C}$-vector spaces on $|\mathscr{S}|$ is called S-constructible if $\left.F\right|_{; \sigma \mid}$ is a constant sheaf for any $\sigma \in \Delta$.

We denote by $\mathrm{S}$-Const $(\mathscr{S})$ the full subcategory of $\operatorname{Mod}(\mathscr{S})$ consisting of all the $\mathrm{S}$-constructible sheaves on $|\mathscr{S}|$.

One can show easily the following lemma.

Lemma 1.2. (i) If $f: F \rightarrow F^{\prime}$ is a homomorphism of $S$-constructible sheaves, then the kernel, the image and the cokernel of $f$ are also S-constructible.

(ii) If $0 \rightarrow F^{\prime} \rightarrow F \rightarrow F^{\prime \prime} \rightarrow 0$ is an exact sequence of sheaves on $|\mathscr{S}|$ and if $F^{\prime}$ and $F^{\prime \prime}$ are $S$-constructible, then $F$ is also $S$-constructible.

Proposition 1.3. Let $F$ be an S-constructible sheaf on $|\mathscr{S}|$. Then, for any $\sigma \in \Delta$ and $x \in|\sigma|$, we have

$$
H^{n}(U(\sigma) ; F)=H^{n}(|\sigma| ; F)=0 \text { for } n \neq 0
$$

and

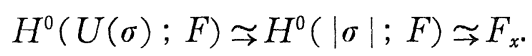

Proof. For $0<\varepsilon<1$, we set $I_{\varepsilon}=\{t \in \mathbb{R} ; \varepsilon \leqq t \leqq 1\}$, and define the map $\pi_{\varepsilon}$ from $I_{\varepsilon} \times U(\sigma)$ into $U(\sigma)$ by; for any $t \in I_{\varepsilon}$ and $y \in U(\sigma)$

$$
\pi_{\varepsilon}(t, y)(p)=t y(p)+(1-t) x(p) \text { for } p \in S \text {. }
$$

Then it is easy to see that $\pi_{\varepsilon}$ is a continuous map from $I_{\varepsilon} \times U(\sigma)$ 
onto $U(\sigma)$ and $\pi_{\varepsilon}(1, y)=y$. Moreover we have $\pi_{\varepsilon}(t, y) \in|\tau|$ for $\tau$ such that $y \in|\tau|$. Thus, if we denote by $h$ the projection from $I_{\varepsilon} \times U(\sigma)$ onto $U(\sigma), \pi_{\varepsilon}^{-1} F$ is a constant sheaf over any fiber of $h$. Hence we have

$$
H^{n}\left(\{\varepsilon\} \times U(\sigma) ; \pi_{\varepsilon}^{-1} F\right) \approx H^{n}\left(I_{\varepsilon} \times U(\sigma) ; \pi_{\varepsilon}^{-1} F\right) \widetilde{\rightarrow} H^{n}\left(\{1\} \times U(\sigma) ; \pi_{\varepsilon}^{-1} F\right) .
$$

On the other hand, $\pi_{\varepsilon}$ is a homeomorphism from $\{1\} \times U(\sigma)$ onto $U(\sigma)$ and hence

$$
H^{n}\left(\{1\} \times U(\sigma) ; \pi_{\varepsilon}^{-1} F\right) \cong H^{n}(U(\sigma) ; F) .
$$

Similarly, we have

$$
H^{n}\left(\{\varepsilon\} \times U(\sigma) ; \pi_{\varepsilon}^{-1} F\right)=H^{n}\left(\pi_{\varepsilon}(\{\varepsilon\} \times U(\sigma)) ; F\right) .
$$

By taking the inductive limit with respect to $\varepsilon$, we obtain

$$
H^{n}(U(\sigma) ; F) \cong \underset{\varepsilon}{\lim } H^{n}\left(\pi_{\varepsilon}(\{\varepsilon\} \times U(\sigma)) ; F\right) .
$$

Remark that $\pi_{\varepsilon}(\{\varepsilon\} \times U(\sigma))$ forms a neighborhood system of $x$. Therefore, the right hand side of (1.2.4) equals $F_{x}$ for $n=0$ and vanishes for $n \neq 0$. Thus we obtain

$$
H^{n}(U(\sigma) ; F)=\left\{\begin{array}{lll}
F_{x} & \text { for } & n=0 \\
0 & \text { for } & n \neq 0
\end{array}\right.
$$

If we apply the same argument to the simplicial complex $\sigma$ and $\left.F\right|_{|\sigma|}$, we obtain

$$
H^{n}(|\sigma| ; F)=\left\{\begin{array}{lll}
F_{x} & \text { for } & n=0 \\
0 & \text { for } & n \neq 0
\end{array}\right.
$$

This proposition particularly implies the following

Q. E. D.

Proposition 1. 4. (i) For any $\sigma, F \mapsto \Gamma(U(\sigma) ; F)$ is an exact functor in $F \in \mathrm{S}-\operatorname{Const}(\mathscr{S})$. (ii) For any $F \in \mathrm{Ob}(\mathrm{S}-\operatorname{Const}(\mathscr{S})$ ), if $F(U(\sigma))=0$ for any $\sigma \in \Delta$, then $F=0$.

1. 3 .

Definition 1. 5. A sheaf $F$ on $|\mathscr{S}|$ is called S-acyclic if

$$
H^{k}(U(\sigma) ; F)=0 \text { for } k \neq 0 \text { and } \sigma \in \Delta .
$$

Then, Proposition 1.3 says that an $\mathrm{S}$-constructible sheaf is $\mathrm{S}$ acyclic. Flabby sheaves are also S-acyclic.

For any sheaf $F$ on $|\mathscr{S}|$, let $\alpha(F)$ denote the sheaf $\bigoplus_{\sigma \in \Delta} F(U(\sigma))_{U(\sigma)}$ *

* For a locally closed set $Z$ of a topological space $X$ and a vector space $V, V_{Z}$ denotes the sheaf on $X$ such that $\left.V_{Z}\right|_{x-Z}=0$ and that $\left.V_{Z}\right|_{z}$ is the constant sheaf on $Z$ with fiber $V$. 
Then we have the canonical homomorphism $t(F): \alpha(F) \rightarrow F$ which is functorial in $F$. Let $G$ be the kernel of $t(F)$ and let $\beta(F)$ denote the cokernel of the composition $\alpha(G) \stackrel{t(G)}{\longrightarrow} G \rightarrow \alpha(F)$. Then we have a homomorphism $s(F): \beta(F) \rightarrow F$ functorial in $F$. It is clear that $\alpha(F)$ and $\beta(F)$ are $\mathrm{S}$-constructible sheaves.

Lemma 1. 6. For any sheaf $F$ and $\sigma \in \Delta$

$$
\Gamma(U(\sigma) ; s(F)): \Gamma(U(\sigma) ; \beta(F)) \rightarrow \Gamma(U(\sigma) ; F)
$$

is an isomorphism.

Proof. Let us consider the diagram

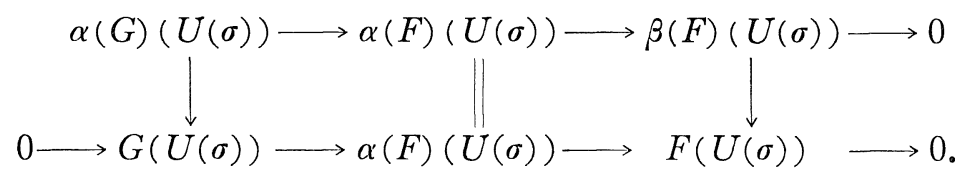

Since $\Gamma(U(\sigma) ; *)$ is an exact functor on $\mathrm{S}$-Const $(\mathscr{S})$, the top row is exact. Since the homomorphism $\alpha(F)(U(\sigma)) \rightarrow F(U(\sigma))$ is surjective, the bottom row is also exact. Then the surjectivity of

$$
\alpha(G)(U(\sigma)) \rightarrow G(U(\sigma))
$$

implies the desired result.

Q. E. D.

Lemma 1.7. If $F$ is $S$-constructible, then $s(F): \beta(F) \rightarrow F$ is an isomorphism.

Proof. Both $F$ and $\beta(F)$ are S-constructible and hence it is sufficient to show that for any $\sigma \in \Delta, \Gamma(U(\sigma) ; s(F))$ is an isomorphism (Proposition 1.4). This is a consequence of the preceding lemma.

Q. E. D.

Lemma 1.6. implies immediately the following

Proposition 1. 8. (i) $\beta$ is a left exact functor from $\operatorname{Mod}(\mathscr{S})$ into $\mathrm{S}-\operatorname{Const}(\mathscr{S})$.

(ii) $\Gamma\left(U(\sigma):\left(R^{k} \beta\right)(F)\right)=H^{k}(U(\sigma) ; F)$ where $R^{k} \beta$ is the k-th right derived functor of $\beta$.

(iii) $R^{k} \beta(F)=0$ for $k \neq 0$ if and only if $F$ is S-acyclic. 
Proof. (i) Let $0 \rightarrow F^{\prime} \rightarrow F \rightarrow F^{\prime \prime} \rightarrow 0$ be an exact sequence in $\operatorname{Mod}(\mathscr{S})$. In order to see that $0 \rightarrow \beta\left(F^{\prime}\right) \rightarrow \beta(F) \rightarrow \beta\left(F^{\prime \prime}\right)$ is exact, it is sufficient to show the exactness of its transform by $\Gamma(U(\sigma) ; *)$ because they are $\mathrm{S}$-constructible sheaves. This transform is, by the preceding lemma, nothing but $0 \rightarrow F^{\prime}(U(\sigma)) \rightarrow F(U(\sigma)) \rightarrow F^{\prime \prime}(U(\sigma))$, which is evidently exact.

(ii) Let $F^{*}$ be an injective resolution of $F$. Then, $R^{k} \beta(F)=\mathscr{H}^{k}$ $\left(\beta\left(F^{*}\right)\right)$. Hence, we have

$$
\begin{aligned}
\Gamma\left(U(\sigma) ; R^{k} \beta(F)\right) & =\Gamma\left(U(\sigma) ; \mathscr{H}^{k}\left(\beta\left(F^{*}\right)\right)\right) \\
& =H^{k}\left(\Gamma\left(U(\sigma) ; \beta\left(F^{*}\right)\right)\right)=H^{k}\left(\Gamma\left(U(\sigma) ; F^{*}\right)\right) \\
& =H^{k}(U(\sigma) ; F) .
\end{aligned}
$$

(iii) This is an immediate consequence of (ii).

Q. E. D.

Proposition 1. 9. Let $F \cdot$ be a complex of $S$-acyclic sheaves satisfying $F^{n}=0$ for $n \ll 0$. If all the cohomology groups of $F^{*}$ are $S$-constructible, then $\beta\left(F^{*}\right) \rightarrow F^{*}$ is a quasi-isomorphism.

Proof. Set $Z^{n}\left(F^{*}\right)=\operatorname{Ker}\left(d_{F}^{n}: F^{n} \rightarrow F^{n+1}\right)$ and $B^{n}\left(F^{*}\right)=\operatorname{Im}\left(d_{F}^{n-1}: F^{n-1}\right.$ $\left.\rightarrow F^{n}\right)$. We shall show by the induction on $n$

$Z^{n}\left(F^{*}\right)$ and $B^{n}\left(F^{*}\right)$ are S-acyclic.

Assume that $Z^{n-1}\left(F^{*}\right)$ and $B^{n-1}\left(F^{*}\right)$ are $\mathrm{S}$-acyclic. We have the exact sequence

$$
0 \rightarrow Z^{n-1}\left(F^{*}\right) \rightarrow F^{n-1} \rightarrow B^{n}\left(F^{*}\right) \rightarrow 0
$$

and

$$
0 \rightarrow B^{n}\left(F^{*}\right) \rightarrow Z^{n}\left(F^{*}\right) \rightarrow H^{n}\left(F^{*}\right) \rightarrow 0 .
$$

The exact sequence (1.3.2) gives the exact sequence

$$
R^{k} \beta\left(F^{n-1}\right) \rightarrow R^{k} \beta\left(B^{n}\left(F^{*}\right)\right) \rightarrow R^{k+1} \beta\left(Z^{n-1}\left(F^{*}\right)\right) .
$$

Since $F^{n-1}$ and $Z^{n-1}\left(F^{*}\right)$ are $\mathrm{S}$-acyclic, this shows $R^{k} \beta\left(B^{n}\left(F^{*}\right)\right)=0$ for $k \neq 0$, which means that $B^{n}\left(F^{*}\right)$ is S-acyclic. In the same way, the exact sequence (1.3.3) implies the S-acyclicity of $Z^{n}\left(F^{*}\right)$ because $B^{n}\left(F^{*}\right)$ and $H^{n}\left(F^{*}\right)$ are S-acyclic. Thus, the induction proceeds and the property (1.3.1) holds for any $n$.

Now the sequence

$$
0 \rightarrow \beta\left(Z^{n}\left(F^{*}\right)\right) \rightarrow \beta\left(F^{n}\right) \rightarrow \beta\left(F^{n+1}\right)
$$


is exact because $\beta$ is left exact. Hence we obtain

$$
\beta\left(Z^{n}\left(F^{*}\right)\right) \cong Z^{n}\left(\beta\left(F^{*}\right)\right) \text {. }
$$

The exact sequence (1.3.2) derives the exact sequence

$$
0 \rightarrow \beta\left(Z^{n-1}\left(F^{*}\right)\right) \rightarrow \beta\left(F^{n-1}\right) \rightarrow \beta\left(B^{n}\left(F^{*}\right)\right) \rightarrow\left(R^{1} \beta\right)\left(Z^{n-1}\left(F^{*}\right)\right),
$$

in which the last term vanishes because of the S-acyclicity of $Z^{n-1}\left(F^{*}\right)$ (see (iii) of Proposition 1.8). Thus (1.3.4) implies

$$
\beta\left(B^{n}\left(F^{*}\right)\right) \cong B^{n}\left(\beta\left(F^{*}\right)\right) .
$$

Finally the exact sequence (1.3.3) gives the exact sequence

$$
0 \rightarrow \beta\left(B^{n}\left(F^{*}\right)\right) \rightarrow \beta\left(Z^{n}\left(F^{*}\right)\right) \rightarrow \beta\left(H^{n}\left(F^{*}\right)\right) \rightarrow 0
$$

because $\left(R^{1} \beta\right)\left(B^{n}\left(F^{*}\right)\right)=0$.

Since $H^{n}\left(F^{*}\right)$ is S-constructible, we have $\beta\left(H^{n}\left(F^{*}\right)\right)=H^{n}\left(F^{*}\right)$ by Lemma 1. 7. This implies the desired result: $H^{n}\left(F^{*}\right) \cong H^{n}\left(\beta\left(F^{*}\right)\right)$.

Q. E. D.

Let us denote by $\mathrm{D}(\mathrm{S}$-Const $(\mathscr{S}))$ the derived category of S-Const $(\mathscr{S})$ and by $\mathrm{D}^{+}(\mathrm{S}$-Const $(\mathscr{S}))$ the full subcategory of $\mathrm{D}(\mathrm{S}$-Const $(\mathscr{S}))$ consisting of $F^{*} \in \mathrm{Ob}\left(\mathrm{D}(\mathrm{S}-\right.$ Const $(\mathscr{S}))$ such that $F^{n}=0$ for $n \ll 0$. Let $\mathrm{D}_{S}^{+}$-const $(\mathscr{S})$ be the full subcategory of the derived category $\mathrm{D}(\operatorname{Mod}$ $(\mathscr{S})$ ) consisting of $F^{*}$ such that $F^{n}=0$ for $n \ll 0$ and that all $H^{n}\left(F^{*}\right)$ are $\mathrm{S}$-constructible. Then the following theorem is an immediate consequence of the preceding proposition.

Theorem 1. 10. $\mathrm{D}^{+}(\mathrm{S}-$ Const $(\mathscr{S}))$ and $\mathrm{D}_{\mathrm{S}-\text { Const }}^{+}(\mathscr{S})$ are equivalent by the canonical functor

$$
\mathrm{D}^{+}(\mathrm{S}-\text { Const }(\mathscr{S})) \rightarrow \mathrm{D}_{\mathrm{S} \text {-Const }}^{+}(\mathscr{S}) \text { given by } F^{*} \longmapsto F^{*}
$$

and $\boldsymbol{R} \beta: \mathrm{D}_{S-\text { Const }}^{+}(\mathscr{S}) \longrightarrow \mathrm{D}^{+}(\mathrm{S}-$ Const $(\mathscr{S}))$.

Remark. The category S-Const $(\mathscr{S})$ is equivalent to the category $\mathscr{A}$ of covariant functors from $\Delta$ into the category of vector spaces. Here $\Delta$ is regarded as the category as follows: $\operatorname{Hom}(\sigma, \tau)=\phi$ if $\sigma \not \subset \tau$ and $\operatorname{Hom}(\sigma, \tau)$ consists of the single element if $\sigma \subset \tau$. The functor from $\mathrm{S}-\operatorname{Const}(\mathscr{S}) \rightarrow \mathscr{A}$ is given by $F \mapsto\{\sigma \mapsto F(U(\sigma))\}$. The converse functor $\mathscr{A} \rightarrow \mathrm{S}$-Const $(\mathscr{S})$ is constructed as follows: for an object $F(\sigma)$ of $\mathscr{A}$, we define two homomorphisms $f$ and $g$ from $\bigoplus_{\tau \supset \sigma} F(\sigma)_{U(\tau)}$ into $\oplus_{\sigma} F(\sigma)_{U(\sigma)}$ by $F(\sigma)_{U(\tau)} \longleftrightarrow F(\sigma)_{U(\sigma)}$ and by $F(\sigma)_{U(\tau)} \rightarrow F(\tau)_{U(\tau)}$, respectively. 
Then, we assign $\operatorname{Coker}(f-g)$ to the functor $F(\sigma)$.

1. 4.

Proposition 1. 11. For any $S$-constructible sheaf $F, \sigma \in \Delta$ and a vector space $V$, we have

$$
\operatorname{Hom}\left(F, V_{\overline{|\overline{\mid}|}}\right)=\operatorname{Hom}_{c}(F(U(\sigma)), V) \text {. }
$$

Here, $\overline{|\sigma|}$ means the closure of $|\sigma|$.

Proof. By Proposition 1. 3, we have $\Gamma\left(U(\sigma) ; V_{|\overline{|\sigma|}|} \cong \Gamma\left(|\sigma| ; V_{|\sigma|}\right) \cong V\right.$. Hence we obtain the canonical homomorphism

$$
\operatorname{Hom}\left(F, V_{\overline{|\sigma|}}\right) \longrightarrow \operatorname{Hom}_{c}(F(U(\sigma)), V) \text {. }
$$

We shall show that this is an isomorphism for any $F$. If $F$ has the form $W_{U(\tau)}$ for a vector space $W$ and $\tau \in \Delta$, then we have

$$
\begin{aligned}
\operatorname{Hom}\left(F, V_{\overline{|\sigma|} \mid}\right) & =\operatorname{Hom}\left(W, \Gamma\left(U(\tau) ; V_{\overline{|\sigma|} \mid}\right)\right) \\
& =\operatorname{Hom}\left(W, \Gamma\left(U(\tau) \cap|\sigma| ; V_{\overline{|\bar{\sigma}|}}\right)\right) .
\end{aligned}
$$

Now, it is easy to see that the following four conditions are equivalent: $\quad U(\tau) \cap|\overline{\sigma \mid} \neq \phi, \quad U(\tau) \supset| \sigma|, \quad| \tau \mid \subset \overline{|\sigma|}$ and $\tau \subset \sigma$. Thus, we obtain

$$
\Gamma\left(U(\tau) \cap|\bar{\sigma}| ; V_{\overline{|\sigma|}}\right)= \begin{cases}V & \text { if } \tau \subset \sigma \\ 0 & \text { otherwise. }\end{cases}
$$

On the other hand, we have

$$
\operatorname{Hom}_{C}(F(U(\sigma)), V)=\operatorname{Hom}_{c}\left(\Gamma\left(U(\sigma) ; W_{U(\tau)}\right), V\right) .
$$

Therefore we have by Proposition 1.3

$$
\Gamma\left(U(\sigma) ; W_{U(\tau)}\right)= \begin{cases}W & \text { if } \tau \subset \sigma \\ 0 & \text { otherwise. }\end{cases}
$$

They imply that (1.4.1) is an isomorphism for any $F$ of the form $W_{U(\tau)}$. Hence for any sheaf $F,(1.3 .1)$ is an isomorphism for $\alpha(F)$. Since there exists an exact sequence, (See Lemma 1.7.)

$$
\alpha(G) \rightarrow \alpha(F) \rightarrow F \rightarrow 0
$$

and the both sides of (1.4.1) is left exact in $F$, we obtain the desired result.

Q. E. D.

Now, for any S-constructible sheaf $F$ we define $\gamma(F)$ by

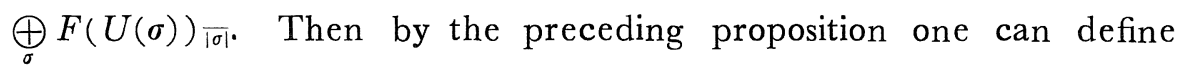
the canonical homomorphism

$$
F \rightarrow \gamma(F)
$$


which is injective.

1. 5. If $\mathscr{S}=(S, \Delta)$ and $\mathscr{S}^{\prime}=\left(S^{\prime}, \Delta^{\prime}\right)$ are two simplicial complex, then a morphism $g$ from $\mathscr{S}$ to $\mathscr{S}^{\prime}$ is by definition a map from $S$ into $S^{\prime}$ such that $g(\sigma) \in \Delta^{\prime}$ for any $\sigma \in \Delta$. For a morphism $g$ from $\mathscr{S}$ to $\mathscr{S}^{\prime}$, one can define a continuous map $|g|$ from $|\mathscr{S}|$ into $\left|\mathscr{S}^{\prime}\right|$ as follows: for any $x \in|\mathscr{S}|,|g|(x)(q)=\sum_{p \in g^{-1}(q)} x(p)$ for $q \in S^{\prime}$. It is easy to see that $|g|(|\sigma|)=|g(\sigma)|$ and $|g|(\mid \overline{|\sigma|})=\overline{|g(\sigma)|}$. Moreover, any fiber of $|\bar{\sigma}| \rightarrow|\overline{g(\sigma)}|$ is contractible.

\section{§2. R-Constructible Sheaves on a Real Analytic Manifold}

2. 1. Let $M$ be a real analytic manifold. In this paper, all real analytic manifolds are assumed paracompact. We shall recall the notion of subanalytic sets due to Hironaka.

Definition 2. 1. A subset $Z$ of $M$ is called subanalytic at a point $\hat{p}$ of $M$ if there exist a neighborhood $W$ of $p$, an integer $r$, real analytic manifolds $N_{j}^{(\nu)}$ and proper real analytic maps $f_{j}^{(\nu)}$ from $N_{j}^{(\nu)}$ into $W(\nu=1,2 ; j=1,2, \cdots, r)$ such that $Z \cap W=\bigcup_{j=1}^{r}\left(f_{j}^{(1)}\left(N_{j}^{(1)}\right)-\right.$ $\left.f_{j}^{(2)}\left(N_{j}^{(2)}\right)\right)$. A subset is called subanalytic if it is subanalytic at any point of $M$.

For the properties of subanalytic sets we refer to [H1] and [H2].

For instance, the following results are known.

Proposition 2. 2. (i) The union and the intersection of a locally finite family of subanalytic subsets are subanalytic.

(ii) The complement of a subanalytic subsets is subanalytic.

(iii) The closure, the boundary and the interior of a subanalytic set are subanalytic.

Proposition 2. 3. A closed subanalytic set is the image of a real analytic manifold by a proper real analytic map.

Proposition 2. 4. A relatively compact subanalytic subsets has only a finite number of connected components and they are subanalytic. 
Proposition 2. 5. Let $\left\{Z_{j}\right\}_{j \in J}$ be a locally finite family of subanalytic subsets of a real analytic manifold $M$ such that $M=\cup Z_{j}$. Then, there exist a simplicial complex $\mathscr{S}=(S, \Delta)$ and a homeomorphism $\iota:|\mathscr{S}| \Im M$ satisfying the following conditions:

(i) For any $\sigma \in \Delta, \iota(|\sigma|)$ is a submanifold of $M$ which is subanalytic.

(ii) For any $\sigma \in \Delta$, there exists $j \in J$ such that $\iota(|\sigma|) \subset Z_{j}$.

\section{2.}

Definition 2. 6. Let $F$ be a sheaf of $\boldsymbol{C}$-vector spaces on a real analytic manifold $M$. We say that $F$ is weakly $\mathbb{R}$-constructible if there exists a locally finite family $\left\{M_{j}\right\}_{j \in J}$ of subanalytic subsets of $M$ such that $\left.F\right|_{M_{j}}$ is a locally constant sheaf on $M_{j}$ for any $j \in J$ and that $M=\cup M_{j}$.

Definition 2. 7. For a weakly $\boldsymbol{R}$-constructible sheaf $F$, we say that $F$ is $\mathbb{R}$-constructible if $\operatorname{dim} F_{x}<\infty$ for any $x \in M$.

Let us denote by $\operatorname{Mod}(M)$ the abelian category of sheaves of $\boldsymbol{C}$-vector spaces on $M$, and by $\boldsymbol{R}$-Const $(M)$ the full subcategory of $\operatorname{Mod}(M)$ consisting of $\boldsymbol{R}$-constructible sheaves on $M$. We shall denote by $\mathrm{D}(M)$ the derived category of $\operatorname{Mod}(M)$ and denote by $\mathrm{D}_{\boldsymbol{R}-\mathrm{c}}^{\mathrm{b}}(M)$ the full subcategory of $\mathrm{D}(M)$ consisting of $F^{*}$ such that $\mathscr{H}^{j}\left(F^{*}\right)$ are $\boldsymbol{R}$-constructible for any $j$ and that $F^{j}=0$ except for a finite many $j$.

Theorem 2. 8. The canonical functor

$$
\mathrm{D}^{\mathrm{b}}(\boldsymbol{R} \text {-Const }(M)) \rightarrow \mathrm{D}_{\boldsymbol{R}-\mathrm{c}}^{\mathrm{b}}(M)
$$

is an equivalence.

Proof. In order to prove this, we have to show the following two statements.

(a) For any $F^{*} \in \mathrm{Ob}\left(\mathrm{D}_{\boldsymbol{R}-c}^{\mathrm{b}}(M)\right)$, there exists $G^{*} \in \mathrm{D}^{\mathrm{b}}(\boldsymbol{R}$-Const $(M))$, such that $G^{*}$ is isomorphic to $F^{*}$ (as an object in $\mathrm{D}_{\boldsymbol{R}-\mathrm{c}}^{\mathrm{b}}(M)$ ).

(b) $\operatorname{Hom}_{D^{\mathrm{b}}(\boldsymbol{R}-\text { Const }(M))}\left(F^{*}, G^{*}\right) \underset{\sim}{\rightarrow} \operatorname{Hom}_{D_{\boldsymbol{R}-\mathrm{c}}^{\mathrm{b}}(M)}\left(F^{*}, G^{*}\right)$

for any $F^{*}, G^{*} \in \mathrm{Ob}\left(\mathrm{D}^{\mathrm{b}}(\boldsymbol{R}\right.$-Const $(M))$.

First we shall prove the statement (a).

Let $F^{*}$ be an object of $\mathrm{D}_{\boldsymbol{R}-\mathrm{c}}^{\mathrm{b}}(M)$. We may assume from the beginning that $F^{n}=0$ for $n \ll 0$ and that all $F^{n}$ are flabby. Since all $\mathscr{H}^{n}\left(F^{*}\right)$ are $\boldsymbol{R}$-constructible and $\mathscr{H}^{n}\left(F^{*}\right)=0$ except for a finite many 
$n$, there exists a locally finite family $\left\{M_{j}\right\}_{j \in J}$ of subanalytic subsets of $M$ such that $M=\cup M_{j}$ and that $\left.\mathscr{H}^{n}\left(F^{*}\right)\right|_{M_{j}}$ are locally constant for any $n$ and any $j \in J$. Hence there exist a simplicial complex $\mathscr{S}=(S, \Delta)$ and a homeomorphism $\iota:|\mathscr{S}| \Im M$ satisfying the following conditions:

(2.2.1) $\quad(|\sigma|)$ is subanalytic for any $\sigma \in \Delta$,

(2.2.2) For any $\sigma \in \Delta$, there exists $j \in J$ such that $\iota(|\sigma|) \subset M_{j}$.

Therefore we have

(2.2.3) $\quad \iota^{-1} \mathscr{H}^{n}\left(F^{*}\right)$ is $S$-constructible for any $n$.

Since $\iota^{-1} F^{n}$ are flabby, Proposition 1. 9 implies that $G^{*}=\beta\left(\iota^{-1} F^{*}\right)$ is quasi-isomorphic to $\iota^{-1} F$. Since $G^{*}$ is a complex of $S$-constructible sheaves, $\iota_{*} G^{*}$ is a complex of $\boldsymbol{R}$-constructible sheaves by (2.2.1). Hence $\iota_{*}\left(G^{*}\right)$ is an object of $D^{b}(\mathbb{R}$-Const $(M))$ which is quasiisomorphic to $F^{*}$. Thus we obtain (a).

Now, we shall prove (b).

For $F^{*}, G^{*} \in \mathrm{Ob}\left(\mathrm{D}^{\mathrm{b}}(\mathbb{R}\right.$-Const $\left.(M))\right)$, there exist a simplicial complex $\mathscr{S}=(S, \Delta)$ and a homeomorphism $\iota:|\mathscr{S}| \widetilde{\rightarrow} M$ satisfying (2.2.1) and the following condition (2.2.4).

(2.2.4) $\iota^{-1} F^{n}, c^{-1} G^{n}$ are $S$-constructible for any $n$.

Then we have the diagram:

(2. 2. 5) $\operatorname{Hom}_{\mathrm{D}\left(\mathrm{S}-\operatorname{Const}\left(\mathscr{S}_{)}\right)\right.}\left(\iota^{-1} F^{*}, \iota^{-1} G^{*}\right) \underset{v}{\longrightarrow} \operatorname{Hom}_{\mathrm{D}(\boldsymbol{R}-\operatorname{Const}(M))}\left(F^{*}, G^{*}\right)$

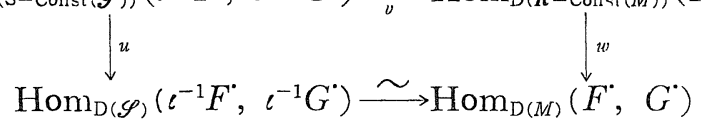

Theorem 1.10 implies that $u$ is an isomorphism. Hence $w$ is surjective.

Now, we shall prove that $w$ is injective. Let $\varphi$ be an element of $\operatorname{Hom}_{\mathrm{D}^{\mathrm{b}}(\boldsymbol{R}-\operatorname{Const}(M))}\left(F^{*}, G^{*}\right)$ such that $w(\varphi)=0$. Hence there exist a quasi-isomorphism $G^{\cdot} \rightarrow G^{\prime}$ and a homomorphism $\varphi^{\prime}: F^{*} \rightarrow G^{\prime}$ of complexes, which give $\varphi$. Hence by replacing $G^{\cdot}$ with $G^{\prime}$, we may assume from the beginning that $\varphi$ is given by a homomorphism of complexes from $F^{*}$ into $G^{*}$, which we shall denote by the same letter $\varphi$. Then, in the diagram (2.2.5), $\varphi=v\left(e^{-1}(\varphi)\right)$ and hence $w(\varphi)=$ $u\left(\iota^{-1} \varphi\right)=0$. This implies $\iota^{-1}(\varphi)=0$ by Theorem 1.10 and finally we obtain $\varphi=v\left(\iota^{-1}(\varphi)\right)=0$. 


\section{§3. Tempered Distributions}

3. 0. In this section, we shall study the properties of the tempered homomorphisms from $\boldsymbol{R}$-constructible sheaves into the sheaf of distributions (in the sense of L. Schwartz).

This notion is a generalization of tempered distributions studied by Martineau [Ma], Lojasiewicz [L], ... An original notion of a tempered distribution is a distribution $u$ on $\boldsymbol{R}^{n}$ which satisfies the condition:

$$
\left|\int u(x) \varphi(x) d x\right| \leqq \sum_{|\alpha| \leqq m} \sup \left|D_{x}^{\alpha} \varphi(x)\right| \text { for any } \varphi \in C_{0}^{\infty}\left(\boldsymbol{R}^{n}\right) .
$$

Here, $C_{0}^{\infty}\left(\boldsymbol{R}^{n}\right)$ denotes the space of $C^{\infty}$-functions on $\boldsymbol{R}^{n}$ with compact support. If we compactify $\mathbb{R}^{n}$ to $S^{n}$ by adding one point, then this notion is equivalent to saying that $u$ is continued to a distribution defined on $S^{n}$.

As a generalization of this, we arrive to the definition of tempered homomorphisms (Def. 3. 13).

3. 1. Let $M$ be a real analytic paracompact manifold, $\mathscr{A}_{M}$ the sheaf of real analytic functions and $\mathscr{D}_{M}$ the sheaf of differential operators of finite order with $\mathscr{A}_{M}$ as coefficients. Let us denote by $\mathscr{D} b_{M}$ the sheaf of distributions on $M$.

Definition 3. 1. A distribution $u$ defined on an open subset $U$ of $M$ is called tempered at a point $p$ of $M$ if there exist a neighborhood $W$ of $p$ and a distribution $v$ defined on $W$ such that $\left.u\right|_{W \cap U}=\left.v\right|_{W \cap U}$. If $u$ is tempered at any point, then we say that $u$ is tempered.

Then one can easily prove the following lemma.

Lemma 3.2. Let $u$ be a distribution defined on an open subset $U$ of $M$. Then the following conditions a re equivalent.

(i) $u$ is tempered.

(ii) $u$ is tempered at any point of $\partial U=\bar{U}-U$.

(iii) There exists a distribution $w$ defined on $M$ such that $u=\left.w\right|_{U}$.

We have also the following lemma. For a subset $A$ of $\boldsymbol{R}^{n}$ and a point $x$ of $\mathbb{R}^{n}$, we denote

$$
\mathrm{d}(x, A)=\inf \{|y-x| ; y \in A\} .
$$


Lemma 3. 3. Let $u(x)$ be a distribution defined on a relatively compact open subset $U$ of $\boldsymbol{R}^{n}$. Then the following conditions are equivalent:

(i) $u$ is tempered (at any point of $\mathbb{R}^{n}$ ).

(ii) There exist a positive $C$ and a positive integer $m$ such that

$$
\left|\int u(x) \varphi(x) d x\right| \leqq C \sum_{|\alpha| \leqq m} \sup \left|D^{\alpha} \varphi\right|
$$

for any $\varphi \in C_{0}^{\infty}(U)$.

(iii) There exist a positive $C$ and positive integers $m$ and $r$ such that

$$
\left|\int u(x) \varphi(x) d x\right| \leqq C \sum_{|\alpha| \leqq m} \sup \left(\mathrm{d}(x, \partial U)^{-r}\left|D^{\alpha} \varphi(x)\right|\right)
$$

for any $\varphi \in C_{0}^{\infty}(U)$.

Proof. (i) $\Rightarrow$ (ii) $\Rightarrow$ (iii) is trivial.

(ii) $\Rightarrow$ (i) is an immediate consequence of Hahn-Banach's extension theorem.

We shall prove (iii) $\Rightarrow$ (ii). Let $\varphi \in C_{0}^{\infty}(U)$ and $x$ a point of $U$. Then there exists $y \in U$ such that supp $\varphi \not \nexists y,|x-y|<\mathrm{d}(x, \partial U)$ and $x+t(y-x) \in U$ for $0 \leqq t \leqq 1$. On the other hand, we have

$$
\varphi(x)=\frac{(-1)^{\nu}}{(\nu-1) !} \int_{0}^{1} t^{\nu-1}\left(\frac{d}{d t}\right)^{\nu} \varphi(x+t(y-x)) d t
$$

for $\nu \geqq 1$.

Hence there exists a constant $C_{\nu}$ which does depend only on $n$ and $\nu$ such that

$$
\begin{aligned}
|\varphi(x)| & \leqq C_{\nu}|y-x|^{\nu} \sum_{|\alpha| \leqq \nu} \sup \left|D^{\alpha} \varphi\right| \\
& \leqq C_{\nu} \mathrm{d}(x, \partial U)^{\nu} \sum_{|\alpha| \leqq \nu} \sup \left|D^{\alpha} \varphi\right| .
\end{aligned}
$$

Applying this to $D^{\alpha} \varphi$ instead of $\varphi$, we obtain

$$
\begin{aligned}
& \mathrm{d}(x, \partial U)^{-\nu}\left|D_{x}^{\alpha} \varphi(x)\right| \leqq C_{\nu} \sum_{|\beta| \leqq \nu+|\alpha|} \sup \left|D^{\beta} \varphi\right| \\
& \text { for } x \in U \text { and } \varphi \in C_{0}^{\infty}(U) .
\end{aligned}
$$

Therefore (3.1.3) implies (3.1.2).

Q.E.D.

3. 2. Lojasiewicz ([L] p. 98, Prop. 5) shows the following theorem.

Theorem 3. 4. Let $M=\mathbb{R}^{n}$ and let $U_{1}, U_{2}$ be two relatively compact open subsets of $M$. Suppose that there exist an open neighborhood $W$ of $\partial\left(U_{1} \cup U_{2}\right)$, a positive constant $C$ and a positive integer $m$ such that 
(3.2.1)

$$
\begin{aligned}
& \mathrm{d}\left(x, \quad \partial U_{1}\right)+\mathrm{d}\left(x, \quad \partial U_{2}\right) \geqq C\left(\mathrm{~d}\left(x, \quad \partial\left(U_{1} \cup U_{2}\right)\right)\right)^{m} \\
& \text { for } x \in W .
\end{aligned}
$$

Then a distribution $u$ defined on $U_{1} \cup U_{2}$ is tempered if and only if $\left.u\right|_{U_{1}}$ and $\left.u\right|_{U_{2}}$ are tempered.

On the other hand, Hironaka proved the following theorem.

Theorem 3.5 ([H2]). Let $A$ and $B$ be two closed subanalytic subsets of $\mathbb{R}^{n}$. Then, for any compact set $K$ there exist a positive integer $m$ and a positive constant $C$ such that

$$
\mathrm{d}(x, A)+\mathrm{d}(x, B) \geqq C(\mathrm{~d}(x, A \cap B))^{m} \text { for } x \in K .
$$

Hence these two theorems imply the following

Theorem 3. 6. Let $U$ be a subanalytic subset of a real analytic manifold $M$ and $\left\{U_{j}\right\}_{j \in J}$ a finite open covering of $U$ by open subanalytic subsets $U_{j}$. Then a distribution $u$ defined on $U$ is tempered if and only if $\left.u\right|_{U_{j}}$ is tempered for any $j \in J$.

By this theorem, we can localize the notion of tempered distribution.

3. 3. Lojasiewicz also proved the following

Lemma 3. 7. (Lojasiewicz's inequality [L]). Let $f(x)$ be a real analytic function defined on an open subset $U$ of $\boldsymbol{R}^{n}$, and let $Z$ denote $f^{-1}(0)$. Then, for any compact subset $K$ of $U$, there exist a positive constant $C$ and a positive integer $m$ such that

$$
|f(x)| \geqq C(\mathrm{~d}(x, Z))^{m} \quad \text { for } x \in K .
$$

This lemma together with Lemma 3. 3 immediately implies the following lemma.

Lemma 3. 8. Let $u$ be a distribution defined on an open subset $U$ of a real analytic manifold $M$ and let $g(x)$ be a real analytic function defined on $M$ which does not vanish at any point of $U$. Then $u$ is tempered if and only if gu is tempered. Assume moreover $g$ is positive-valued on $U$. Then for any $\lambda \in C, g^{\lambda} u$ is tempered if and only if so is $u$.

Now we shall prove the following 
Proposition 3.9. Let $f: M \rightarrow N$ be a real analytic map from a real analytic manifold $M$ into a real analytic manifold $N$. Let $U$ be an open subset of $M$ such that $f$ induces a submersion from $U$ into $N$. Then for any distribution $u$ defined on $N,\left.\left(f^{*} u\right)\right|_{U}$ is a tempered distribution.

Proof. The question being local we may assume that $M$ is an open subset of $\boldsymbol{R}^{m}$ and $N$ is an open subset of $\mathbb{R}^{n}$. We can assume also $m \geqq n$. Set $Z=\{z \in M ; \operatorname{rank}(\mathrm{d} f)(x)<n\}$. Then we can assume $U=M-Z$. Let us take a point $p$ of $M$ and consider all the problems on a neighborhood of $p$. Then there exist a finite number of real analytic maps $g_{i}: M \rightarrow \mathbb{R}^{m-n}$ such that $U=\cup U_{i}$, where $U_{i}=\{x \in U$; rank $\left.\mathrm{d} f_{i}(x)=m\right\}$, and $f_{i}=\left(g_{i}, f\right): M \rightarrow \mathbb{R}^{m-n} \times N$. If we denote by $p$ the projection from $\boldsymbol{R}^{m-n} \times N$ onto $N$, and if we set $v=\left.\left(f^{*} u\right)\right|_{U}$, then we have $\left.v\right|_{U_{i}}=\left.f_{i}^{*}\left(p^{*} u\right)\right|_{U_{i}}$. Since all $U_{i}$ are subanalytic, $v$ is tempered if $\left.f_{i}^{*}\left(p^{*} u\right)\right|_{U_{i}}$ is tempered for any $i$. Hence, by replacing $N, f, u$ and $U$ with $\mathbb{R}^{m-n} \times N, f_{\imath}, p^{*} u$ and $U_{\imath}$, respectively, we may assume from the beginning $m=n$ and $U=M-Z$. Let $g(x)$ be the Jacobian $\partial y / \partial x$ of $f(x)$. Then $\left.g\right|_{Z}=0$ and $g(x) \neq 0$ for $x \in M-Z$. Therefore there exists an integer $l$ such that

$$
|g(x)| \geqq \text { const. }(\mathrm{d}(x, Z))^{l} \text { for } \quad x \in M \text {. }
$$

Let us denote by $x=\left(x_{1}, \ldots, x_{n}\right)$ (resp. $y=\left(y_{1}, \ldots, y_{n}\right)$ ) points of $M$ (resp. $N)$. Then there exists real analytic functions $h_{i, j}(x) \quad(1 \leqq i$, $j \leqq n)$ such that

$$
\begin{aligned}
& g(x) \frac{\partial}{\partial y_{i}}=\sum_{j=1}^{n} h_{i, j}(x) \frac{\partial}{\partial x_{j}} \quad(1 \leqq i \leqq n) \\
& \text { by } y=f(x) .
\end{aligned}
$$

Hence $g^{2|\alpha|} D_{y}^{\alpha}$ is a linear combination of $D_{x}^{\beta}(|\beta| \leqq|\alpha|)$ with real analytic functions in $y$ as coefficients. Note that $u(y)$ satisfies

$$
\left|\int u(y) \varphi(y) d y\right| \leqq \text { Const. } \sum_{|\alpha| \leqq m} \sup \left|D_{y}^{\alpha} \varphi\right| \text { for } \varphi \in C_{0}^{\infty}(N)
$$

by shrinking $N$ if necessary.

For $\phi(x) \in C_{0}^{\infty}(U)$,

$$
\int_{U}\left(f^{*} u\right)(x) \phi(x) d x=\int_{N} u(y) \phi\left(f^{-1}(y)\right)\left|g\left(f^{-1}(y)\right)^{-1}\right| d y
$$

where $g(x)$ is the Jacobian $y=f(x)$. Hence we have 


$$
\begin{aligned}
& \left|\int\left(f^{*} u\right)(x) \phi(x) d x\right| \\
& \quad \leqq \operatorname{const} \sum_{|\alpha| \leqq m} \sup \left|D_{y}^{\alpha}\left(\phi\left(f^{-1}(y)\right) g\left(f^{-1}(y)\right)^{-1}\right)\right| \\
& \quad \leqq \operatorname{const} \sum_{|\alpha| \leqq m} \sup g(x)^{-2|\alpha|}\left|D_{x}^{\alpha}\left(\phi(x) g(x)^{-1}\right)\right| \\
& \quad \leqq \operatorname{const} \sum_{|\alpha| \leqq m} \sup g(x)^{-3|\alpha|-1}\left|D_{x}^{\alpha} \psi(x)\right| .
\end{aligned}
$$

Hence we obtain

$$
\begin{aligned}
& \left|\int\left(f^{*} u\right)(x) \psi(x) d x\right| \leqq \text { const } \sum_{|\alpha| \leqq m} \sup \mathrm{d}(x, Z)^{-(3|\alpha|+1) l}\left|D_{x}^{\alpha} \psi(x)\right| \\
& \quad \text { for any } \phi \in C_{0}^{\infty}(U) .
\end{aligned}
$$

Hence it follows from Lemma 3.3 that $\left.\left(f^{*} u\right)\right|_{U}$ is tempered.

Q. E. D.

Proposition 3. 10. Let $f: M \rightarrow N$ be a proper real analytic map. Let $U$ be an open subset of $N$ such that $f^{-1}(U) \rightarrow U$ is an isomorphism. Then a distribution $u$ defined on $U$ is tempered if and only if $\left.\left(f^{*} u\right)\right|_{f^{-1}(U)}$ is tempered.

Proof. The question being local in $N$, we may assume that $N$ is an open subset of $\boldsymbol{R}^{n}$. If $u$ is tempered then there exists a distribution $\tilde{u}$ defined on $N$ such that $\left.\tilde{u}\right|_{U}=u$. Hence, $\left.\left(f^{*} u\right)\right|_{f^{-1}(U)}=$ $\left.\left(f^{*} \tilde{u}\right)\right|_{f^{-1}(U)}$ is a tempered distribution by the preceding lemma. Conversely assume that $\left.f^{*} u\right|_{f^{-1} U}$ is tempered. Let us fix nowhere vanishing real analytic densities $\mu_{M}$ and $\mu_{N}$ on $M$ and $N$, respectively. Set $g=f^{*} \mu_{N} / \mu_{M}$. Then $g$ is a real analytic function which does not vanish on $f^{-1} U$. Therefore, $\left.g^{-1}\left(f^{*} u\right)\right|_{f^{-1} U}$ is also tempered by Lemma 3. 8. Let $w$ be a distribution defined on $M$ which extends $g^{-1}\left(\left.f^{*} u\right|_{f^{-1} U}\right)$. We define the distribution $w^{\prime}$ on $N$ by

$$
\int_{N} w^{\prime} \varphi \mu_{N}=\int_{M} w(\varphi \circ f) \mu_{M} \text { for } \varphi \in C_{0}^{\infty}(N) \text {. }
$$

Then it is easy to see that $\left.w^{\prime}\right|_{U}=u$.

Q. E. D.

Remark 3. 11. In L. Schwartz [S], a distribution $u(x)$ defined on $\boldsymbol{R}^{n}$ is called tempered if

$$
\left|\int u(x) \varphi(x) d x\right| \leqq C \sum_{|\alpha| \leqq m} \sup \left|D^{\alpha} \varphi\right| \text { for any } \varphi \in C_{0}^{\infty}\left(\boldsymbol{R}^{n}\right) .
$$

This condition is equivalent to saying that there is a distribution $\tilde{u}$ 
defined on $\mathbb{P}^{n}(\boldsymbol{R})$ such that $\left.\tilde{u}\right|_{\mathbb{R}^{n}}=u$; i. e. $u$ is tempered in $\mathbb{P}^{n}(\mathbb{R})$.

Remark 3. 12. This notion of tempered distributions have been studied by several mathematicians. See $[\mathrm{Ma}],[\mathrm{L}]$.

3. 4. Let $M$ be a paracompact real analytic manifold.

Definition 3. 13. For an $\boldsymbol{R}$-constructible sheaf $F$ defined on $M$, $T-\mathscr{H} O m\left(F, \mathscr{D} b_{M}\right)$ is the subsheaf of $\mathscr{H o m}_{C}\left(F, \mathscr{D}_{b_{M}}\right)$ defined as follows: for any open subset $U$ of $M$

$$
\begin{aligned}
& \Gamma\left(U ; T-\mathscr{H} o m\left(F, \mathscr{D} b_{M}\right)\right)=\left\{\varphi \in \Gamma\left(U ; \mathscr{H} o m_{c}\left(F, \mathscr{D} b_{M}\right)\right) ;\right. \\
&\varphi \text { satisfies the following condition (3. 4. 1) }\}
\end{aligned}
$$

(3.4.1) For any relatively compact open subanalytic subset $V$ of $U$ and $s \in F(V), \varphi(s)$ is a tempered distribution.

Similarly, for any locally free $\mathscr{A}_{M}$-Module $\mathscr{V}$ of finite rank, we can define $T$ - $\mathscr{H} o m\left(F, \mathscr{D} \mathfrak{b}_{M} \otimes_{\mathscr{A}_{M}} \mathscr{V}\right)$ and we have

$$
T-\mathscr{H} o m\left(F, \mathscr{D}_{\mathfrak{b}_{M}} \otimes_{\mathscr{A}_{M}} \mathscr{V}\right)=T-\mathscr{H} o m\left(F, \mathscr{D} \mathfrak{b}_{M}\right) \bigotimes_{\mathscr{Z}_{M}} \mathscr{V}
$$

Note that $T-\mathscr{H} o m\left(F, \mathscr{D} \ell_{M}\right)$ is a sheaf of $\mathscr{D}_{M}$-modules. Hereafter, for the sake of simplicity, we write $T H(F)$ for $T-\mathscr{H} o m\left(F, \mathscr{D} 6_{M}\right)$. If we want to emphasize the manifold $M$, we write $T H_{M}(F)$ for it.

Since $T H_{M}(F)$ is a sheaf of modules over the ring of $C^{\infty}$-functions, we have

Proposition 3. 14. For any $\mathbb{R}$-constructible sheaf $F, T H(F)$ is a soft sheaf.

Lemma 3. 15. Let $U$ be a subanalytic open subset of $M$ and $u$ a tempered distribution defined on $U$. Then the homomorthism $\varphi$ from $\boldsymbol{C}_{U}$ to $\mathscr{D} b_{M}$ defined by $1_{U} \mapsto u$ belongs to $\Gamma\left(M ; T H\left(\mathbb{C}_{U}\right)\right)$. Here $1_{U}$ signifies the element of $\Gamma\left(U ; \mathbb{C}_{U}\right)$ which corresponds to the constant function with value 1 .

Proof. Let $V$ be a relatively compact subanalytic open subset of $M$ and let $s$ be a section of $\boldsymbol{C}_{U}$ over $V$. We have to prove that $\varphi(s)$ is a tempered distribution. As mentioned in Proposition 2. 4, $V$ has 
finitely many connected components $V_{1}, \ldots, V_{l}$. Then $s$ is a constant function on each $V_{j}$ because $\boldsymbol{C}_{U} \subset \boldsymbol{C}_{X}$. Since Supp $s \subset U,\left.s\right|_{V_{j}}=0$ if $V_{j} \not \subset U$. Hence $\left.\varphi(s)\right|_{V_{j}}$ is a tempered distribution if $V_{j} \not \subset U$. If $V_{j} \subset U,\left.\varphi(s)\right|_{v_{j}}$ is a constant multiple of $\left.u\right|_{V_{j}}$ and hence tempered. In both cases, $\left.\varphi(s)\right|_{v_{j}}$ is tempered and hence $\varphi(s)$ is tempered by Theorem 3. 6 . Q. E. D.

This lemma implies the following corollary.

Corollary 3. 16. For any open subanalytic subset $U$ and an open subset $\Omega$ of $M$, we have

$$
\begin{aligned}
\Gamma\left(\Omega ; T H\left(\boldsymbol{C}_{U}\right)\right)= & \left\{u \in \Gamma\left(U \cap \Omega ; \mathscr{D} b_{M}\right) ; u\right. \text { is tempered at } \\
& \text { any point of } \Omega\} .
\end{aligned}
$$

Corollary 3. 17. For any closed subanalytic subset $Z$ of $M$, we have

$$
T H\left(\boldsymbol{C}_{Z}\right)=\Gamma_{Z}(\mathscr{D} 6) \text {. }
$$

If $\operatorname{Mod}\left(\mathscr{D}_{M}\right)$ denotes the category of $\mathscr{D}_{M}$-Modules, then $T H(*)$ is a contravariant functor from $\boldsymbol{R}$-Const $(M)$ into $\operatorname{Mod}\left(\mathscr{D}_{M}\right)$. This functor enjoys the following remarkable property.

Theorem 3. 18. $T H(*)$ is an exact functor from $\boldsymbol{R}$-Const $(M)$ into $\operatorname{Mod}\left(\mathscr{D}_{M}\right)$.

Proof. This theorem follows from the following two lemmas.

Lemma 3. 19. Let $\varphi: F \rightarrow G$ be a surjective homomorphism of $\boldsymbol{R}$-constructible sheaves $F$ and $G$ and let $\psi$ be a section of $\mathscr{H}$ om( $G$, $\left.\mathscr{D} k_{M}\right)$. If $\psi \circ \varphi$ belongs to $T H(F)$, then $\phi$ belongs to $T H(G)$.

Lemma 3. 20. Let $\varphi: F \rightarrow G$ be an injective homomorphism of $\boldsymbol{R}$-constructible sheaves $F$ and $G$. Then $T H(G) \rightarrow T H(F)$ is surjective.

In order to prove Lemma 3. 19 we prepare the following lemma.

Lemma 3. 21. Let $\varphi: F \rightarrow G$ be as in Lemma 3. 19. Then, for any relatively compact open subanalytic set $V$ and any $s$ in $G(V)$, there exist a finite open covering $\left\{V_{j}\right\}_{j \in J}$ of $V$ by subanalytic sets $V_{j}$ and elements $t_{j}$ of $F\left(V_{j}\right)$ such that $\varphi\left(t_{j}\right)=\left.s\right|_{V_{j}}$. 
Proof. By Proposition 2. 5, there exist a simplicial complex $\mathscr{S}=$ $(S, \Delta)$ and a homeomorphism $\iota:|\mathscr{S}| \widetilde{\rightarrow} M$ satisfying

(3.4.2) $\quad(|\sigma|)$ is subanalytic for any $\sigma \in \Delta$.

(3.4.3) $\iota^{-1} F$ and $\iota^{-1} G$ are $S$-constructible.

(3.4.4) For any $\sigma \in \Delta$, we have either $\iota(|\sigma|) \subset V$ or $\iota(|\sigma|) \cap V=\dot{\phi}$.

Then $\Delta^{\prime}=\left\{\sigma \in \Delta ;|\sigma| \subset \iota^{-1} V\right\}$ is a finite set and $e^{-1} V=\bigcup_{\sigma \in \Delta^{\prime}} U(\sigma)$. Then the lemma follows from the surjectivity of the homomorphisms $\Gamma\left(U(\sigma) ; \iota^{-1} F\right) \rightarrow \Gamma\left(U(\sigma) ; \iota^{-1} G\right)$.

Q. E. D.

Now, we shall prove Lemma 3.19. Let $\phi$ be a section of $\mathscr{H o m}\left(F, \mathscr{D} b_{M}\right)$ over an open subset $U$ of $M$ such that $\phi \circ \varphi$ belongs to $T H(F)$. Let $V$ be a relatively compact, open, subanalytic subset of $U$ and let $s$ be a section of $G$ over $V$. Then, the preceding lemma assures the existence of a finite open covering $\left\{V_{j}\right\}$ of $V$ by subanalytic sets $V_{j}$ and $t_{j} \in F\left(V_{j}\right)$ such that $\left.s\right|_{V_{j}}=\varphi\left(t_{j}\right)$. Since $\phi \circ \varphi$ belongs to $T H(F),\left.\phi(s)\right|_{V_{j}}=\psi\left(\varphi\left(t_{j}\right)\right)$ is tempered for any $j$. Hence $\phi(s)$ is tempered by Theorem 3.6. Thus Lemma 3. 19 is proved.

Next we shall prove Lemma 3. 20. In order to prove this lemma, it is sufficient to show the surjectivity of the map $\varphi^{*}: \Gamma(M ; T H(G))$ $\rightarrow \Gamma(M ; T H(F))$. Let $\phi$ be an element of $\Gamma(M ; T H(F))$. Let us take a simplicial complex $\mathscr{S}=(S, \Delta)$ and a homeomorphism $\iota:|\mathscr{S}| \rightarrow$ $M$ satisfying (3.4.2) and (3.4.3). Let $\mathscr{A}$ be the set of $\left(F^{\prime}, \psi^{\prime}\right)$, where $F^{\prime}$ is a subsheaf of $G$ such that $\iota^{-1} F^{\prime}$ is $S$-constructible and $\psi^{\prime}$ is an element of $\Gamma\left(M ; T H\left(F^{\prime}\right)\right)$. We introduce the order on $\mathscr{A}$ as follows: $\left(F^{\prime}, \phi^{\prime}\right)<\left(F^{\prime \prime}, \phi^{\prime \prime}\right)$ if and only if $F^{\prime \prime} \supset F^{\prime}$ and $\psi^{\prime}=\left.\psi^{\prime \prime}\right|_{F^{\prime}}$

We shall prove first that $\mathscr{A}$ is inductively ordered. Let $\mathscr{A}^{\prime}=$ $\left\{\left(F_{\lambda}, \phi_{\lambda}\right)\right\}$ be a linearly ordered subset of $\mathscr{A}$. Set $F^{\prime}=\cup F_{\lambda}$. Then, it is clear that $\iota^{-1} F^{\prime}$ is an $S$-constructible sheaf, and there exists a unique element $\phi^{\prime} \in \Gamma\left(M ; \mathscr{H o m}\left(F^{\prime}, \mathscr{D} k_{M}\right)\right)$ such that $\left.\phi^{\prime}\right|_{F_{\lambda}}=\phi_{\lambda}$. In order to prove that $\phi^{\prime}$ is in $T H\left(F^{\prime}\right)$, let $s$ be a section over a relatively compact, open, subanalytic subset $V$ of $M$. Then for any $\sigma \in \Delta^{\prime}=\left\{\sigma \in \Delta ;|\sigma| \cap \iota^{-1} V \neq \phi\right\},|\sigma| \cap \iota^{-1} V$ has only finite many connected components. Moreover, $\left.F_{\lambda}\right|_{\epsilon(|\sigma|)}$ is a constant sheaf. Therefore we have $\Gamma\left(\iota(|\sigma|) \cap V ; F^{\prime}\right)=\bigcup \Gamma\left(\iota(|\sigma|) \cap V ; F_{\lambda}\right)$. Since $\Delta^{\prime}$ is a finite set, there exists $\lambda$ such that $\left.{ }^{\lambda}\right|_{\iota(|\sigma|) \cap V} \in \Gamma\left(\iota(|\sigma|) \cap V ; F_{\lambda}\right)$ for any $\sigma \in \Delta^{\prime}$. 
This implies that $s \in \Gamma\left(V ; F_{\lambda}\right)$ for such a $\lambda$. Therefore $\phi^{\prime}(s)=\phi_{\lambda}(s)$ is a tempered distribution. This shows that $\psi^{\prime}$ belongs to $T H\left(F^{\prime}\right)$.

This $\left(F^{\prime}, \phi^{\prime}\right)$ is clearly a supremum of $\mathscr{A}^{\prime}$ and hence $\mathscr{A}$ is inductively ordered. Therefore by Zorn's lemma there exists a maximal element $\left(F^{\prime}, \phi^{\prime}\right)$ of $\mathscr{A}$ such that $\left(F^{\prime}, \phi^{\prime}\right) \succ(F, \phi)$.

In order to prove Lemma 3.20, it is sufficient to show $G=F^{\prime}$. For this purpose, we shall prove $F^{\prime}(\iota(U(\sigma)))=G(\iota(U(\sigma)))$ for any $\sigma$. Let $s$ be an element of $G(\iota(U(\sigma)))$.

Let $\bar{s}$ be the section of $G / F^{\prime}$ over $\iota(U(\sigma))$ which corresponds to $s$ and let $Z$ be the support of $\bar{s}$. Then we have the exact sequence

$$
0 \longrightarrow C_{\iota(U(\sigma))-Z} \stackrel{\alpha}{\longrightarrow} F^{\prime} \oplus \boldsymbol{C}_{\iota(U(\sigma))} \longrightarrow G
$$

where $\boldsymbol{C}_{\iota(U(\sigma))-Z} \rightarrow F^{\prime}$ is given by $\left.s\right|_{\iota(U(\sigma))-Z}$ and $\boldsymbol{C}_{\iota(U(\sigma))-Z} \rightarrow \boldsymbol{C}_{\iota(U(\sigma))}$ is the canonical injection. Then the distribution $u=\psi^{\prime}\left(\left.s\right|_{\ell(U(\sigma))-Z}\right)$ is a tempered distribution, and hence there exists a tempered distribution $\tilde{u}$ defined on $\iota(U(\sigma))$ such that $\left.\tilde{u}\right|_{\iota(U(\sigma))-Z}=-u$. Then $\phi: \boldsymbol{C}_{U(\sigma)} \ni 1 \mapsto \tilde{u}$ belongs to $T H\left(\boldsymbol{C}_{U(\sigma)}\right)$ by Corollary 3. 16. Let $\xi$ be the homomorphism $\left(\varphi^{\prime}, \phi\right)$ from $F^{\prime} \oplus \boldsymbol{C}_{U(\sigma)}$ into $\mathscr{D} b_{M}$. Then $\xi$ belongs to $\Gamma\left(M ; T H\left(F^{\prime} \oplus\right.\right.$ $\left.\left.\boldsymbol{C}_{U(\sigma)}\right)\right)$ and satisfies $\xi \circ \alpha=0$. Let $F^{\prime \prime}$ be the cokernel of $\alpha$. Then $\xi$ gives the homomorphism $\psi^{\prime \prime}$ from $F^{\prime \prime}$ into $\mathscr{D} \mathfrak{l}_{M}$. Lemma 3. 19 implies that $\phi^{\prime \prime}$ belongs to $T H\left(F^{\prime \prime}\right)$, and hence $\left(F^{\prime \prime}, \psi^{\prime \prime}\right)$ is an element of $\mathscr{A}$ such that $\left(F^{\prime \prime}, \phi^{\prime \prime}\right)>\left(F^{\prime}, \phi^{\prime}\right)$. The maximality of $\left(F^{\prime}, \phi^{\prime}\right)$ implies $F^{\prime}=F^{\prime \prime}$. This shows that $Z=\phi$, which implies $s \in F^{\prime}(\iota(U(\sigma)))$. Thus we have proved $F^{\prime}(\iota(U(\sigma)))=G(\iota(U(\sigma)))$ for any $\sigma \in \Delta$. Then $G=F^{\prime}$ follows from Proposition 1.4. This completes the proof of Lemma 3. 20, and also the proof of Theorem 3. 18.

Proposition 3. 22. If $Z$ is a closed subanalytic subset of $M$, and if $F$ is an $\boldsymbol{R}$-constructible sheaf on $M$, then we have

$$
\Gamma_{Z}(T H(F))=T H\left(F_{Z}\right) .
$$

Proof. Let us consider the exact sequence

$$
0 \rightarrow F_{X-Z} \rightarrow F \rightarrow F_{Z} \rightarrow 0 .
$$

Thus we obtain an exact sequence

$$
0 \rightarrow T H\left(F_{Z}\right) \rightarrow T H(F) \rightarrow T H\left(F_{X-Z}\right) .
$$

By taking the functor $\Gamma_{Z}$, this gives the exact sequence 


$$
0 \rightarrow T H\left(F_{Z}\right) \rightarrow \Gamma_{Z}(T H(F)) \rightarrow \Gamma_{Z}\left(T H\left(F_{X-Z}\right)\right) .
$$

On the other hand, we have

$$
\Gamma_{Z}\left(T H\left(F_{X-Z}\right)\right) \subset \Gamma_{Z} \mathscr{H o m}\left(F_{X-Z} ; \mathscr{D} l_{M}\right)=\mathscr{H} o m\left(\left(F_{X-Z}\right)_{Z} ; \mathscr{D} l_{M}\right)=0 .
$$

Thus we have the desired result.

Q. E. D.

Proposition 3. 23. Let $f$ be a real analytic function on $M$ and $F$ an $\mathbb{R}$-constructible sheaf on $M$. Set $U=M-f^{-1}(0)$. Then,

$$
T H(F)_{f} \rightarrow T H\left(F_{U}\right)
$$

is an isomorphism. Here $*_{f}$ means the localization by $f$.

Proof. The multiplication map by $f$ on $T H\left(F_{U}\right)$ is evidently bijective and hence the homomorphism $T H(F) \rightarrow T H\left(F_{U}\right)$ decomposes

$$
T H(F) \rightarrow T H(F)_{f} \rightarrow T H\left(F_{U}\right) .
$$

Since $T H(F) \rightarrow T H\left(F_{U}\right)$ is surjective, $T H(F)_{f} \rightarrow T H\left(F_{U}\right)$ is surjective.

Now, we shall prove that $T H(F)_{f} \rightarrow T H\left(F_{U}\right)$ is bijective. Since $T H$ is an exact functor, we may assume without loss of generality, that $F$ has the form $\mathbb{C}_{Z}$ for a closed subanalytic set $Z$. In this case, the proposition follows from the fact that for any distribution $u$ supported on $f^{-1}(0)$ is annihilated by a power of $f$. E. D.

3. 5. We shall denote by $\mathrm{D}\left(\mathscr{D}_{M}\right)$ the derived category of the category $\operatorname{Mod}\left(\mathscr{D}_{M}\right)$ of $\mathscr{D}_{M}$-Modules. Since $T H(*)$ is an exact contravariant functor from $\mathbb{R}$-Const $(M)$ into $\operatorname{Mod}\left(\mathscr{D}_{M}\right)$, this gives a contravariant functor

$$
\mathbb{R} T H: \mathrm{D}^{\mathrm{b}}(\mathbb{R} \text {-Const }(M)) \rightarrow \mathrm{D}\left(\mathscr{D}_{M}\right) .
$$

By Theorem 2. 8, $\mathrm{D}^{\mathrm{b}}(\boldsymbol{R}$-Const $(M))$ is equivalent to $\mathrm{D}_{\boldsymbol{R}-\mathrm{c}}^{\mathrm{b}}(M)$. Thus we obtain the functor

$$
\mathbb{R} T H: \mathrm{D}_{R-c}^{\mathrm{b}}(M) \rightarrow \mathrm{D}\left(\mathscr{D}_{M}\right) \text {. }
$$

By this construction, for any $F^{*} \in \mathrm{Ob}\left(\mathrm{D}_{\boldsymbol{R}-\mathrm{c}}^{\mathrm{b}}(M)\right), \mathbb{R} T H\left(F^{*}\right)=T H\left(G^{*}\right)$, where $G^{*}$ is a bounded complex of $\mathbb{R}$-constructible sheaves which is quasi-isomorphic to $F^{\circ}$.

When we want to emphasize the manifold $M$, we shall write $\mathbb{R} T H_{M}(*)$ for $\mathbb{R} T H(*)$. 


\section{§4. Functorial Properties for $T H(*)$}

4. 0. In this section, we shall investigate the relation between $T H_{N}$ (*) and $T H_{M}(*)$ for a real analytic map $f: N \rightarrow M$.

4. 1. We shall denote by $\omega_{M}$ the sheaf of orientation on $M$. Hence, for a connected, orientable open subset $U$ of $M$, we have $\Gamma\left(U ; \omega_{M}\right)$ $\cong \boldsymbol{Z}$. This isomorphism depends on the choice of orientation and changes its sign if we change the orientation of $U$. We shall denote by $\Omega_{M}^{p}$ the sheaf of $p$-forms with real analytic coefficients and put $\mathscr{V}_{M}^{(p)}=\Omega_{M}^{n+p} \bigotimes_{Z} \omega_{M}$. Set $\mathscr{V}_{M}=\mathscr{V}_{M}^{(0)}=\Omega_{M}^{n} \bigotimes_{Z} \omega_{M}$. The sheaf $\mathscr{V}_{M}$ is called the sheaf of densities with real analytic coefficients. If we denote by $\mathscr{C}_{M}^{\infty}$ the sheaf of $C^{\infty}$-functions on $M$, then $\mathscr{C}_{M}^{\infty} \bigotimes_{\mathscr{Q}_{M}}^{\infty} \mathscr{V}_{M}$ is the sheaf of densities with $C^{\infty}$-coefficients.

By this notation, a distribution is a continuous functional on $\Gamma_{c}\left(M ; \mathscr{C}_{M}^{\infty} \stackrel{\mathscr{A}}{M}_{M} \otimes \mathscr{V}_{M}\right)$ and a section of $\mathscr{D}_{\mathscr{C}_{M}} \otimes_{\mathscr{A}_{M}} \mathscr{V}_{M}$ is a continuous functional on $\Gamma_{c}\left(M ; \mathscr{C}_{M}^{\infty}\right)$ with the appropriate topology.

4. 2. Let $f: M \rightarrow N$ be a real analytic map. For any sheaf $\mathscr{F}$ on $M$, we shall denote by $f_{1}(\mathscr{F})$ the direct image with proper support; i. e. for any open subset $U$ of $N$,

(4.2.1) $\Gamma\left(U ; f_{1}(\mathscr{F})\right)=\left\{s \in \Gamma\left(f^{-1}(U) ; \mathscr{F}\right)\right.$; supp $s$ is proper over $\left.U\right\}$.

Now, we shall define the integration map

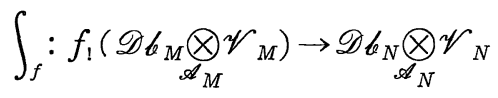

by $\left\langle\int_{f} u, \varphi\right\rangle=\langle u, \varphi \circ f\rangle$ for $\varphi \in C_{c}^{\infty}(U)$ and $u \in \Gamma\left(U ; f_{1}\left(\mathscr{D} b_{M} \otimes_{\mathscr{A}_{M}} \mathscr{V}_{M}\right)\right)$.

Note that supp $u \cap \operatorname{supp} \varphi \circ f$ is a compact subset in $f^{-1} U$. This homomorphism is $\mathscr{A}_{N}$-linear. By tensoring $\mathscr{V}_{N}^{\otimes-1}$, we obtain

$$
\int_{f}: f_{1}\left(\mathscr{D} \mathfrak{b}_{M} \otimes \mathscr{A}_{M} \mathscr{V}_{M / N}\right) \rightarrow \mathscr{D} \mathfrak{b}_{N}
$$

where $\mathscr{V}_{M / N}=\mathscr{V}_{M} \otimes \mathscr{V}_{N}^{\otimes(-1)}$.

Note that the composition

$$
f_{1}\left(\mathscr{D} b_{M} \otimes_{\mathscr{A}_{M}} \mathscr{V}_{M}^{(-1)}\right) \stackrel{d}{\longrightarrow} f_{1}\left(\mathscr{D} b_{M} \otimes_{\mathscr{Z}_{M}} \mathscr{V}_{M}\right) \stackrel{\int_{f}}{\longrightarrow} \mathscr{D} b_{N} \otimes \mathscr{V}_{N}
$$

vanishes. In fact, for $s \in \Gamma\left(U ; f_{1}\left(\mathscr{D} b_{M} \otimes \mathscr{V}_{M}^{(-1)}\right)\right)$ and $\varphi \in C_{0}^{\infty}(U)$, we have $\left\langle\int_{f} \mathrm{~d} s, \varphi\right\rangle=\langle\mathrm{d} s, \varphi \circ f\rangle=-\langle s, \mathrm{~d}(\varphi \circ f)\rangle=0$. 
4. 3. Let $f: M \rightarrow N$ be a real analytic map, and let $\mathscr{D}_{M}$ and $\mathscr{D}_{N}$ denote the sheaf of differential operators of finite order with real analytic coefficients on $M$ and $N$, respectively. Then, we define

$$
\mathscr{D}_{M \rightarrow N}=\mathscr{A}_{M} \bigotimes_{f^{-1} \mathscr{A}_{N}} f^{-1} \mathscr{D}_{N}
$$

and

$$
\mathscr{D}_{N \leftarrow M}=f^{-1} \mathscr{D}_{N} \otimes_{f^{-1} \mathscr{A}_{N}{ }_{N}} \mathscr{V}_{M / N}
$$

where $\mathscr{V}_{M / N}=\mathscr{V}_{M} \bigotimes_{f^{-1} \mathscr{A}_{N}} f^{-1}\left(\mathscr{V}_{N}^{\otimes-1}\right)$.

Then one can define the canonical homomorphisms

$$
\mathscr{D}_{M \rightarrow N} \bigotimes_{f^{-1} \mathscr{D}_{N}} f^{-1} \mathscr{A}_{N} \rightarrow \mathscr{A}_{M}
$$

and

$$
f^{-1} \mathscr{V}_{N} \underset{f^{-1} \mathscr{D}_{N}}{\otimes} \mathscr{D}_{N \leftarrow M} \rightarrow \mathscr{V}_{M}
$$

Moreover, we can endow the structure of $\left(\mathscr{D}_{M}, f^{-1} \mathscr{D}_{N}\right)$-bi-Module on $\mathscr{D}_{M \rightarrow N}$ and that of $\left(f^{-1} \mathscr{D}_{N}, \mathscr{D}_{M}\right)$-bi-Module on $\mathscr{D}_{N \leftarrow M}$ so that (4.3.3) and (4.3.4) are $\mathscr{D}_{M}$-linear (See [K2]).

With these notations, one can state the main theorem of this section.

Theorem 4. 1. Let $f: M \rightarrow N$ be a real analytic map and $F^{*} \in$ $\mathrm{D}_{\boldsymbol{R}-\mathrm{c}}^{\mathrm{b}}(M)$. Assume the following conditions.

(4.3.5) $\mathscr{H}^{j}\left(F^{*}\right)$ is of finite rank for any $j$.

(4.3.6) The closure of the support of $\mathscr{H}^{j}\left(F^{*}\right)$ is proper over $N$ for any $j$.

Then we have a canonical isomorphism in $\mathrm{D}\left(\mathscr{D}_{N}\right)$ :

$$
\boldsymbol{R} f_{*}\left(\mathscr{D}_{N \leftarrow M}{\underset{\mathscr{D}}{M}}_{\mathbb{L}}^{\boldsymbol{R}} T H_{M}\left(F^{*}\right)\right) \simeq \boldsymbol{R} T H_{N}\left(\boldsymbol{R} f_{*} F^{*}\right) .
$$

Here $\bigotimes_{\mathscr{D}_{M}}^{L}$ denotes the left derived functor of $\underset{\mathscr{D}_{M}}{\otimes}$.

The proof of this theorem will be given in $\$ 4.5-\S 4.11$ in three steps. In $\S 4.5$, we treat the case when $f$ is an embedding and in $\S 4.6-\S 4.10$ the case when $f$ is of maximal rank. Finally we shall complete the proof of Theorem 4.1 in $\$ 4.11$.

4. 4. Before entering into the proof of the theorem, we shall remark the following thing. If we denote by $\mathscr{B}$ the sheaf of hyperfunctions 
and replace the functor $T H(*)$ with $\mathscr{H} o m_{C}(*, \mathscr{B})$ in the statement of the theorem, then one obtains the following statement.

$$
\begin{aligned}
\boldsymbol{R} f_{*} & \left(\mathscr{D}_{N \leftarrow M} \stackrel{\mathscr{D}}{\otimes}_{M}^{\boldsymbol{R}} \mathscr{H o m}_{\boldsymbol{C}}\left(F^{*}, \mathscr{B}_{M}\right)\right) \\
\simeq & \mathbb{R} \mathscr{H} o m_{\boldsymbol{C}}\left(\boldsymbol{R} f_{*}\left(F^{*}\right), \mathscr{B}_{N}\right) .
\end{aligned}
$$

It turns out that this is also true by Poincaré duality provided that $f$ is smooth. In fact, one can prove that $\mathscr{D}_{N \leftarrow M} \stackrel{L}{\bigotimes} \underset{\mathscr{D}_{M}}{\mathscr{B}_{M}} \cong f^{-1} \mathscr{B}_{N} \bigotimes_{Z}$ $\omega_{M / N}[l]$ where $l=\operatorname{dim} M-\operatorname{dim} N$ and $\omega_{M / N}=\omega_{M} \otimes \omega_{N}^{\otimes-1}$. Since $\mathscr{B}_{M}$ is a flabby sheaf (and hence an injective object in $\operatorname{Mod}(M)$ ), we obtain

$$
\mathscr{D}_{N \leftarrow M}{\underset{\mathscr{D}}{M}}_{\mathbb{L}}^{L} \boldsymbol{R}{\mathscr{H} o m_{C}}\left(F^{*}, \mathscr{B}_{M}\right)=\boldsymbol{R}{\mathscr{H} o m_{C}}\left(F^{*}, f^{-1} \mathscr{B}_{M} \bigotimes_{Z} \omega_{M / N}[l]\right) \text {. }
$$

Then (4.4.1) follows from Poincaré duality (Verdier [V]).

Therefore, the difficulty to prove the theorem lies on the fact that $\mathscr{D} b$ is not a flabby sheaf.

4. 5. We shall prove Theorem 4.1 when $f$ is a closed embedding. In this case, $\mathscr{D}_{N \leftarrow M}$ is a free $\mathscr{D}_{M}$-Module, and hence it is sufficient to prove

$$
f_{*}\left(\mathscr{D}_{N \leftarrow M} \otimes_{\mathscr{D}_{M}} T H_{M}(F)\right) \cong T H_{N}\left(f_{*} F\right)
$$

for an $\boldsymbol{R}$-constructible sheaf $F$ of finite rank on $M$.

Lemma 4.2. If $M$ is a closed submanifold of $N$ then $\Gamma_{M}\left(\mathscr{D} b_{N}\right) \cong$ $\mathscr{D}_{N \leftarrow M} \otimes \mathscr{D}_{M} \mathfrak{l}_{M}$.

Proof. Take a local coordinate system $(x, y)=\left(x_{1}, \ldots, x_{n}, y_{1}, \ldots\right.$, $\left.y_{m}\right)$ of $N$ such that $M$ is given by $x=0$. Then $\mathscr{D}_{N \leftarrow M}$ is a free $\mathscr{D}_{M^{-}}$ Module generated by $D_{x}^{\alpha} \otimes|d x|^{\otimes-1}\left(\alpha=\left(\alpha_{1}, \ldots, \alpha_{n}\right) \in \mathbb{Z}_{+}^{n}\right)$.

On the other hand, it is well-known that any distribution $u(x, y)$ supported on $M$ can be uniquely written in the form

$$
u(x, y)=\sum D_{x}^{\alpha} \delta(x) u_{\alpha}(y) .
$$

Hence $\sum\left(D_{x}^{\alpha} \otimes|d x|^{-1}\right) \otimes u_{\alpha}(y) \leftrightarrow \sum D_{x}^{\alpha} \delta(x) u_{\alpha}(y)$ gives the isomorphism between $\mathscr{D}_{N \leftarrow M} \otimes_{\mathscr{D}_{M}} \mathscr{D}_{k_{M}}$ and $\Gamma_{M}\left(\mathscr{D} b_{N}\right)$.

Now, we shall prove (4.5.1) when $f$ is a closed embedding. We have a series of homomorphisms 


$$
\begin{aligned}
& f_{*}\left(\mathscr{D}_{N \leftarrow M} \otimes T \mathscr{D}_{M}(F)\right) \rightarrow f_{*}\left(\mathscr{D}_{N \leftarrow M} \otimes \mathscr{H}_{\mathscr{D}_{M}} \mathscr{H}_{C}\left(F, \mathscr{D} k_{M}\right)\right) \\
& \rightarrow f_{*} \mathscr{H o m}_{C}\left(F, \mathscr{D}_{N \leftarrow M} \otimes_{\mathscr{D}_{M}} \mathscr{D} k_{M}\right) \Im f_{*} \mathscr{H o m}_{C}\left(F, f^{-1} \Gamma_{M}\left(\mathscr{D} b_{N}\right)\right) \\
& \rightarrow \mathscr{H o m} c\left(f_{*} F, \mathscr{D} k_{N}\right) \text {. }
\end{aligned}
$$

It is obvious that the image of this composition is contained in $T H_{N}\left(f_{*} F\right)$ and hence we obtain the canonical homomorphism

$$
f_{*}\left(\mathscr{D}_{N \leftarrow M} \underset{\mathscr{D}_{M}}{\otimes} T H_{M}(F)\right) \rightarrow T H_{N}\left(f_{*} F\right) .
$$

Note that the both hand sides are exact in $F$.

The following lemma is almost obvious by the triangulation theorem and we omit the proof.

Lemma 4. 3. For any $\mathbb{R}$-constructible sheaf $F$ of finite rank, there exists a filtration $F=F_{0} \supset F_{1} \supset \cdots$ of $F$ by $\mathbb{R}$-constructible sheaves $\left\{F_{j}\right\}$ satisfying the following condition.

(4.5.3) For any point $x$ of $M$, there exist a neighborhood $U$ of $x$ and $j$ such that $\left.F_{j}\right|_{U}=0$.

(4.5.4) For any $j$, there exists a locally closed subanalytic subset $Z_{j}$ such that $F_{j} / F_{j+1} \cong \mathbb{C}_{Z_{j}}$.

By this lemma, it is sufficient to show that $(4.5 .2)$ is an isomorphism for $F=C_{Z}$ with a subanalytic, locally closed subset $Z$. By the exact sequence $0 \rightarrow \boldsymbol{C}_{Z} \rightarrow \boldsymbol{C}_{\bar{Z}} \rightarrow \boldsymbol{C}_{\partial Z} \rightarrow 0$, we may assume further that $Z$ is a closed subanalytic subset of $M$. In this case, we have $T H_{M}\left(\boldsymbol{C}_{Z}\right)=\Gamma_{Z}\left(\mathscr{D} b_{M}\right)$ and $T H_{N}\left(f_{*} F\right)=\Gamma_{f(Z)}\left(\mathscr{D} b_{N}\right)=\Gamma_{f(Z)}\left(\Gamma_{M}\left(\mathscr{D} b_{N}\right)\right)=$ $\Gamma_{f(Z)}\left(f_{*}\left(\mathscr{D}_{N \leftarrow M} \otimes \mathscr{D}_{M} b_{M}\right)\right)$. They imply immediately that $(4.5 .2)$ is an isomorphism.

4. 6. Next, we shall investigate the case where $f$ is a smooth map (i. e. $d f: T_{x} M \rightarrow T_{x} N$ is surjective for any $x \in M$ ). In this case, $\mathscr{D}_{N \leftarrow M}$ is a right coherent $\mathscr{D}_{M}$-Module and $\mathscr{D}_{N \leftarrow M}$ has a locally free resolution (4.6.1) $\quad 0 \leftarrow \mathscr{D}_{N \leftarrow M} \leftarrow \mathscr{V}_{M / N} \bigotimes_{\mathscr{I}_{M}} \mathscr{D}_{M} \stackrel{d}{\leftarrow} \mathscr{V}_{M / N}^{(-1)} \bigotimes_{\mathscr{A}_{M}} \mathscr{D}_{M} \leftarrow \cdots \stackrel{d}{\leftarrow} \mathscr{V}_{M / N}^{(-l)} \bigotimes_{\mathscr{A}_{M}} \mathscr{D}_{M} \leftarrow 0$. Here $\mathscr{V}_{M / N}^{(-p)}=\Omega_{M / N}^{l-p} \bigotimes_{Z} \omega_{M / N}$ and $l$ is the fiber dimension of $f$. The differential $d$ is given by $d(\omega \otimes P)=d \omega \otimes P+\sum_{j} d x_{j} \omega \otimes \frac{\partial}{\partial x_{j}} P$ for $\omega \in \mathscr{V}_{M / N}^{(-p)}$ and $P \in \mathscr{D}_{M}$. Here we take a local coordinate system $\left(x_{1}, \ldots, x_{n}\right)$ of $M$ and we identify $\mathscr{V}_{M / N}^{(-p)}=\left(\Omega_{M}^{l-p} / \Omega_{M}^{l-p-1} \Omega_{N}^{1}\right) \underset{Z}{\bigotimes_{M / N}}$. 
Hence, for $F^{*} \in \mathrm{D}^{\mathrm{b}}(\boldsymbol{R}$-Const $(M)), \quad \mathscr{D}_{N \leftarrow M} \otimes_{\mathscr{D}_{M}} \boldsymbol{R} T H_{M}\left(F^{*}\right) \quad$ is $\quad$ quasiisomorphic to the simple complex associated with the double complex $\mathscr{V}_{M / N}^{(\cdot)} \bigotimes T H_{M}\left(F^{*}\right)$. Since $\mathscr{V}_{M / N}^{(-p)} \bigotimes_{\mathscr{A}_{M}} T H_{M}\left(F^{j}\right)$ are soft sheaves, $\boldsymbol{R} f_{*}\left(\mathscr{D}_{N \leftarrow M}\right.$ $\left.\bigotimes_{\mathscr{D}_{M}}^{\boldsymbol{L}} \boldsymbol{R} T H_{M}^{\mathscr{A}_{M}}\left(F^{*}\right)\right)$ is isomorphic to $f_{*}\left(\mathscr{V}_{M / N}^{(\cdot)} \bigotimes_{\mathscr{A}_{M}} T H_{M}\left(F^{*}\right)\right)$.

4. 7. For any $\boldsymbol{R}$-constructible sheaf $F$ such that $\overline{\operatorname{supp} F}$ is proper over $N$,

we can define

$$
\begin{aligned}
& \int_{f}: f_{*}\left(\mathscr{V}_{M / N} \otimes T H_{M}(F)\right) \rightarrow \mathscr{H} o m\left(f_{!} F, \mathscr{D}_{b_{N}}\right) \\
& \text { by }\left(\int_{f} \varphi\right)(s)=\int_{f} \varphi(s) \text { for } s \in \Gamma\left(U ; f_{!} F\right) \text { and } \\
& \varphi \in \Gamma\left(U ; f_{*}\left(\mathscr{V}_{M / N} \otimes T H_{M}(F)\right)\right) .
\end{aligned}
$$

It is easy to see that the image is contained in $T H_{N}\left(f_{!} F\right)$ and hence we obtain the homomorphism

$$
\int_{f}: f_{*}\left(\mathscr{V}_{M / N} \otimes T H_{M}(F)\right) \rightarrow T H_{N}\left(f_{!} F\right) \text {. }
$$

By the integration by parts, it is easy to see that the composition

$$
f_{*}\left(\mathscr{V}_{M / N}^{(-1)} \bigotimes_{\mathscr{A}_{M}} T H_{M}(F)\right) \stackrel{d}{\longrightarrow} f_{*}\left(\mathscr{V}_{M / N} \bigotimes T \mathscr{A}_{M} T H_{M}(F)\right) \stackrel{\int_{f}}{\longrightarrow} T H_{N}\left(f_{!} F\right)
$$

vanishes (see §4.2), and one obtains the homomorphism

$$
f_{*}\left(\mathscr{V}_{M / N}^{(\cdot)} \bigotimes_{\mathscr{A}_{M}} T H_{M}(F)\right) \rightarrow T H_{N}\left(f_{!} F\right) \text {. }
$$

If $F^{*} \in \mathrm{D}^{\mathrm{b}}(\boldsymbol{R}$-Const $(M))$ satisfies the condition

(4. 7.2) $\overline{\operatorname{Supp} F^{j}}$ is proper over $N$ for any $j$,

then one has the canonical homomorphism

$$
\int_{f}: f_{*}\left(\mathscr{V}_{M}^{(\cdot)} \otimes_{\mathscr{A}_{M}} \otimes T H_{M}\left(F^{*}\right)\right) \rightarrow T H_{N}\left(f_{:} F^{*}\right) \text {. }
$$

4. 8. Now, we shall admit the following lemma, whose proof will be given in $\S 4.13$.

Lemma 4. 4. For any $F^{*} \in \mathrm{D}^{\mathrm{b}}(\boldsymbol{R}$-Const $(M))$, satisfying (4.7.2), there exist an object $G^{*}$ of $\mathrm{D}(\boldsymbol{R}$-Const $(M))$ and a quasi-isomorphism $F^{*} \rightarrow G^{*}$ such that

(4. 8. 1) $\overline{\text { Supp } G^{j}}$ is proper over $N$ for any $j$. 
(4. 8. 2) For any $j$, there exists a locally finite family $\Delta_{j}$ of closed subanalytic subsets of $M$ such that

$$
G^{j} \cong \bigoplus_{Z \in \Delta_{j}} \boldsymbol{C}_{Z}^{m_{j}(Z)} \quad\left(m_{j}(Z) \in \boldsymbol{Z}_{+}\right)
$$

(4. 8. 2. 2) For any $Z \in \Delta_{j}$, any fiber of $Z \rightarrow f(Z)$ is contractible.

In particular, we have

$$
R^{k} f_{*}\left(G^{j}\right)=0 \text { for any } k \neq 0 \text { and any } j .
$$

If we take such a $G^{*}$ and a quasi-isomorphism $F^{*} \rightarrow G^{*}$, then we have $\boldsymbol{R} f_{*}\left(F^{*}\right) \widetilde{R} f_{*}\left(G^{*}\right) \approx f_{*} G^{*}$. Thus we obtain the homomorphism

$$
\int_{f}: \boldsymbol{R} f_{*}\left(\mathscr{D}_{N \leftarrow M} \mathbb{D}_{\mathscr{D}_{M}}^{\boldsymbol{L}} \boldsymbol{R} T H_{M}\left(F^{*}\right)\right) \rightarrow \boldsymbol{R} T H_{N}\left(\boldsymbol{R} f_{*}\left(F^{*}\right)\right)
$$

as the composition of

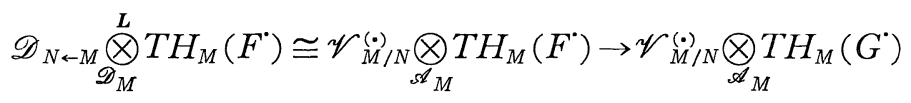

and $\quad \int_{f}: f_{!}\left(\mathscr{V}_{\mathscr{M}^{\prime} / N}^{(\cdot)} \otimes_{\mathscr{A}_{M}} T H_{M}\left(G^{*}\right)\right) \rightarrow T H_{N}\left(f_{!} G^{*}\right)$.

It is a routine to show that thus obtained homomorphism (4.8.4) does not depend on the choice of $G^{*}$ and a quasi-isomorphism $F^{*} \rightarrow G^{*}$, and we omit the proof.

4. 9. We shall prove that (4.8.4) is a quasi-isomorphism. In order to see this, we shall reduce the problem to a special case. For this purpose we remark the following things.

(a) The question is local in $N$.

(b) The question is local in $M$ in the following sense. Any $F^{*} \in$ $\mathrm{D}^{\mathrm{b}}(\boldsymbol{R}$-Const $(M))$, has a filtration such that the support of the graduation is as small as we want.

(c) If $f$ is a composition of two smooth map $g: M \rightarrow L$ and $h: L \rightarrow N$, then $\int_{f}=\int_{h} \cdot \int_{g} \cdot$ More precisely, $\int_{f}: \boldsymbol{R} f_{*}\left(\mathscr{D}_{N \leftarrow M} \stackrel{L}{\otimes} T H_{M}\left(F^{*}\right)\right) \rightarrow$ $\boldsymbol{R} T H_{N}\left(\boldsymbol{R} f_{*} F^{*}\right)$ is the composition of

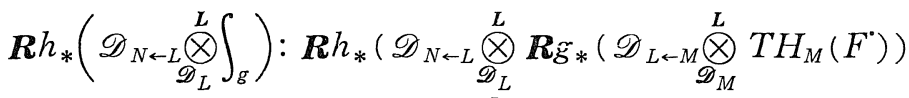

$$
\begin{aligned}
& \rightarrow \boldsymbol{R} h_{*}\left(\mathscr{D}_{N \leftarrow L} \bigotimes_{\mathscr{D}_{L}}^{L} \boldsymbol{R} T H_{L}\left(\boldsymbol{R} g_{*} F^{*}\right)\right)
\end{aligned}
$$

and

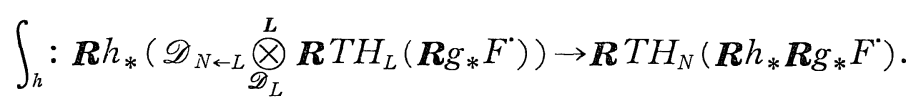


Note that $g^{-1} \mathscr{D}_{N \leftarrow L} \bigotimes_{\mathscr{D}_{L}}^{L} \mathscr{D}_{L \leftarrow M} \cong \mathscr{D}_{N \leftarrow M}$ ([K2] Lemma 4.7). We omit the proof of this.

By these three remarks we can reduce the problem when $M=$ $\boldsymbol{R} \times N$ and $f$ is the projection.

4. 10. Now, we shall prove that (4.8.4) is an isomorphism when $M=\boldsymbol{R} \times N$ and $f$ is the projection. By Lemma 4.4, we may assume that $F=C_{Z}$ for a closed subanalytic subset $Z$ of $M$ such that $Z \cap f^{-1}(x)$ is contractible for any $x \in f(Z)$. Now, we may assume further $Z \subset\{t ;|t| \leqq 1\} \times N \subset M$. Set

$Z_{ \pm}=\{(t, x) \in M ;|t| \leqq 1$ and there exists $s \in \boldsymbol{R}$ such that $(s, x) \in Z$ and $\pm t \geqq \pm s\}$.

Then we have $Z_{+} \cap Z_{-}=Z$, and $Z_{+} \cup Z_{-}=[0,1] \times f(Z)$. Hence, in order to show that (4.8.4) is isomorphic for $F=C_{Z}$, it is sufficient to prove this for $F=C_{Z_{+}}, C_{Z_{-}}$and $C_{Z_{+} \cup Z_{-}}$. Hence, we may assume further that $Z \supset\{0\} \times f(Z)$. Thus, the problem is reduce to the following lemma.

Lemma 4.5. Let $Z$ be a closed, subanalytic subset of $M=\boldsymbol{R} \times N$ satisfying

(4.10.1) $Z \cap f^{-1}(x)$ is contractible for any $x \in f(Z)$.

(4. 10.2) $Z \supset\{0\} \times f(Z)$

(4. 10.3) $Z \subset[-1,1] \times f(Z)$.

Then

$$
0 \longrightarrow f_{*} \Gamma_{Z}\left(\mathscr{D} k_{M}\right) \stackrel{\partial / \partial t}{\longrightarrow} f_{*} \Gamma_{Z}\left(\mathscr{D} k_{M}\right) \stackrel{\int d t}{\longrightarrow} \Gamma_{f(Z)}\left(\mathscr{D} k_{N}\right) \rightarrow 0
$$

is an exact sequence.

Proof. If $u \in \Gamma\left(U ; f_{*} \Gamma_{Z}\left(\mathscr{D} k_{M}\right)\right)$ satisfies $\partial u / \partial t=0$, then $u$ is constant along the fiber. Hence supp $u \subset Z$ implies $u=0$. If $u \in \Gamma(U$; $\left.f_{*} \Gamma_{Z}\left(\mathscr{D} b_{M}\right)\right)$ satisfies $\int u d t=0$, then we define $v$ by the equation

$$
\frac{\partial v}{\partial t}=u,\left.\quad v\right|_{t=-2}=0 \text { 。 }
$$

Then $v$ is constant in $t$ outside $Z$, and $\int u d t=v(t, x)$ for $t>1$. Hence, $v(t, x)=0$ if $|t|>1$. This implies supp $v \subset Z$. Finally we have $\int w(x) \delta(t) d t=w(x)$ for $w \in \Gamma_{f(Z)}\left(\mathscr{D} b_{N}\right)$ Q. E. D. 
Thus Theorem 4.1 is proved when $f$ is smooth or a closed embedding.

4. 11. Now, we shall prove Theorem 4.1 in the general case. Setting $L=N \times M, f$ decomposes into $h \circ g$ where $g: M \rightarrow L$ is the closed embedding of $M$ onto the graph of $f$ and $h$ is the second projection from $L$ to $M$. Hence, we have

$$
\begin{aligned}
& \mathbb{R} f_{*}\left(\mathscr{D}_{N \leftarrow M} \stackrel{L}{\bigotimes}_{\mathscr{D}_{M}}^{L} \mathbb{R} T H_{M}\left(F^{*}\right)\right) \\
& \cong \mathbb{R} h_{*} \boldsymbol{R} g_{*}\left(g^{-1} \mathscr{D}_{N \leftarrow L} \underset{g^{-1} \mathscr{D}_{L}}{\stackrel{L}{\bigotimes}} \mathscr{D}_{L \leftarrow M} \stackrel{\bigotimes}{\mathscr{D}} M^{L} \mathbb{R} T H_{M}\left(F^{*}\right)\right)
\end{aligned}
$$

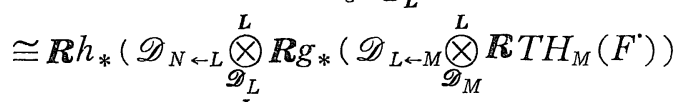

$$
\begin{aligned}
& \cong \mathbb{R} h_{*}\left(\mathscr{D}_{N \leftarrow L} \mathscr{\mathscr { D }}_{L}^{L} \boldsymbol{R} T H_{L}\left(\mathbb{R} g_{*} F^{*}\right)\right) \\
& \cong \boldsymbol{R} T H_{N}\left(\mathbb{R} h_{*} \mathbb{R} g_{*} F^{*}\right) \cong \mathbb{R} T H_{N}\left(\mathbb{R} f_{*} F^{*}\right) \text {. }
\end{aligned}
$$

This completes the proof of Theorem 4.1.

4. 12. As an application of Theorem 4.1, we shall prove the following proposition.

Proposition 4. 6. For $F^{*} \in \mathrm{D}_{R-\mathrm{c}}^{\mathrm{b}}(M)$, we have

$$
\boldsymbol{R} \mathscr{H}_{0} m_{\mathscr{D}_{M}}\left(\mathscr{A}_{M}, \boldsymbol{R} T H_{M}\left(F^{*}\right)\right) \cong \boldsymbol{R} \mathscr{H}_{0} m_{\boldsymbol{C}}\left(F^{*}, \boldsymbol{C}_{M}\right) \text {. }
$$

First remark that if we replace $\mathbb{R} T H_{M}\left(F^{*}\right)$ with $\mathbb{R} \mathscr{H} o m_{C}\left(F^{*}, \mathscr{B}_{M}\right)$ then the proposition is obvious, because $\mathbb{R} \mathscr{H}_{o} m_{\mathscr{D}_{M}}\left(\mathscr{A}_{M}, \mathscr{B}_{M}\right)=\mathbb{C}_{M}$ by Poincaré Lemma and $\mathscr{B}_{M}$ is a flabby sheaf. Hence, it is sufficient to prove that the homomorphism

$$
\text { (4.12.1) } \mathbb{R} \mathscr{H o m}_{\mathscr{D}_{M}}\left(\mathscr{A}_{M}, \operatorname{TH}_{M}(F)\right) \rightarrow \mathbb{R}_{\mathscr{H}} \mathscr{H}_{\mathscr{D}_{M}}\left(\mathscr{A}_{M}, \mathscr{H o m}\left(F, \mathscr{B}_{M}\right)\right)
$$

is an isomorphism for any $\mathbb{R}$-constructible sheaf $F$.

We may assume that $M$ is oriented. Let us take a point $x$ of $M$, and we shall prove that

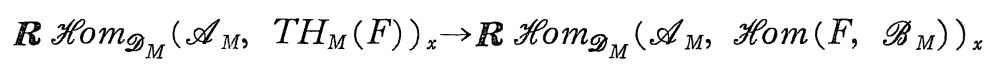

is a quasi-isomorphism. Let $f$ be the map from $M$ onto the manifold $p t$ consisting of the single point. Then, for a relatively compact open subanalytic neighborhood $U$ of $x$, we have

$$
\mathbb{R} \Gamma\left(M ; \mathscr{D}_{p t<M} \bigotimes_{\mathscr{D}_{M}}^{\perp} T H_{M}\left(F_{U}\right)\right)=\operatorname{Hom}_{C}\left(\mathbb{R} \Gamma\left(M, F_{U}\right) ; \mathbb{C}\right)
$$


by Theorem 4.1. On the other hand

$$
\mathscr{D}_{p t \leftarrow M}=\boldsymbol{R} \mathscr{H}_{0 m_{\mathscr{D}_{M}}}\left(\mathscr{A}_{M}, \mathscr{D}_{M}\right)[n] \quad(n=\operatorname{dim} M) .
$$

Hence we obtain

$$
\boldsymbol{R} \Gamma\left(M ; \boldsymbol{R} \mathscr{H} m_{\mathscr{D}_{M}}\left(\mathscr{A}_{M}, T H\left(F_{U}\right)\right) \cong \operatorname{Hom}_{C}\left(\boldsymbol{R} \Gamma_{c}(U ; F), \boldsymbol{C}\right)[n] .\right.
$$

On the other hand, this is true for $\mathscr{H} o m(F, \mathscr{B})$, i. e.

$$
\boldsymbol{R} \Gamma\left(M ; \boldsymbol{R} \mathscr{H o m}_{\mathscr{D}_{M}}\left(\mathscr{A}_{M}, \mathscr{H} o m\left(F_{U}, \mathscr{B}_{M}\right)\right) \cong \operatorname{Hom}_{c}\left(\boldsymbol{R} \Gamma_{c}(U ; F), \boldsymbol{C}\right)[n]\right. \text {. }
$$

Thus we obtain

$\boldsymbol{R} \Gamma\left(M ; \boldsymbol{R} \mathscr{H}_{\mathscr{D}_{\mathscr{D}_{M}}}\left(\mathscr{A}_{M}, T H\left(F_{U}\right)\right) \simeq \boldsymbol{R} \Gamma\left(U ; \boldsymbol{R} \mathscr{H o m}_{\mathscr{D}_{M}}\left(\mathscr{A}_{M}, \boldsymbol{R} \mathscr{H} o m(F\right.\right.\right.$, $\left.\left.\mathscr{B}_{M}\right)\right)$ ).

For $U \fallingdotseq V \ni x$, we have

$\boldsymbol{R} \Gamma\left(M ; \boldsymbol{R} \mathscr{H o m}_{\mathscr{D}_{M}}\left(\mathscr{A}_{M}, T H\left(F_{U}\right)\right) \rightarrow \boldsymbol{R} \Gamma\left(U ; \boldsymbol{R} \mathscr{H o m}_{\mathscr{D}_{M}}\left(\mathscr{A}_{M}, T H(F)\right)\right)\right.$

$\rightarrow \boldsymbol{R} \Gamma\left(M ; \boldsymbol{R} \mathscr{H}_{0} m_{\mathscr{D}_{M}}\left(\mathscr{A}_{M}, T H\left(F_{V}\right)\right)\right)$.

Hence

$$
\begin{aligned}
& \lim _{\vec{U}} \boldsymbol{R} \Gamma\left(M ; \boldsymbol{R} \mathscr{H} m_{\mathscr{Q}_{M}}\left(\mathscr{A}_{M}, T H\left(F_{U}\right)\right)\right) \\
& \quad \cong \lim _{\vec{U}} \boldsymbol{R} \Gamma\left(U ; \boldsymbol{R} \mathscr{H} m_{\mathscr{D}_{M}}\left(\mathscr{A}_{M}, T H(F)\right)\right) .
\end{aligned}
$$

Thus we obtain the desired result.

Q.E. D.

4. 13. Now, we shall prove Lemma 4.4. This is a corollary of $\S 1.4, \S 1.5$ and the following theorem (Hironaka $[\mathrm{H}]$ ).

Theorem 4. 7. Let $f: M \rightarrow N$ be a real analytic map, $K$ a compact subset of $M$ and $\left\{Z_{j}\right\}$ a locally finite covering of $M$ by a locally closed subanalytic subsets $Z_{j}$. Then there exist simplicial complexes $\mathscr{S}=(S, \Delta), \mathscr{S}^{\prime}=\left(S^{\prime}, \Delta^{\prime}\right)$ and a morphism $g: \mathscr{S} \rightarrow \mathscr{S}^{\prime}$, a homeomorphism ' from $|\mathscr{S}|$ onto a neighborhood of $K$ and a homeomorphism i' from $\left|\mathscr{S}^{\prime}\right|$ onto an open subset of $N$ satisfying the following conditions

(4.13.1) $\quad f \circ \iota=\iota^{\prime} \circ|g|$

(4.13.2) For any $\sigma \in \Delta, \iota(|\sigma|)$ is a subanalytic set contained in some $Z_{j}$.

\section{§5. Regular Holonomic Systems}

5. 1. In this section, we shall review the results on regular holono- 
mic systems obtained in $[\mathrm{KK}],[\mathrm{K}]$.

Let $X$ be a complex manifold and $\mathcal{O}_{X}$ the sheaf of holomorphic functions on $X$. Let $\mathscr{D}_{X}(m)$ denote the sheaf of linear differential operators of order at most $m$ with $\mathscr{O}_{X}$ as coefficients, and $\mathscr{D}_{X}=$ $\cup \mathscr{D}_{X}(m)$ the sheaf of linear differential operators. Then the cotangent bundle $T^{*} X$ of $X$ coincides with $\operatorname{Specan}\left(\operatorname{gr} \mathscr{D}_{X}\right)$, where $\operatorname{gr} \mathscr{D}_{X}=\bigoplus_{m} \mathscr{D}_{X}(m) / \mathscr{D}_{X}(m-1)$.

For a $\mathscr{D}_{X}$-Module $\mathscr{M}$, an increasing sequence $\left\{\mathscr{M}_{j}\right\}_{j \in Z}$ of coherent $\mathcal{O}_{X^{-}}$-sub-Modules of $\mathscr{M}$ is called a good filtration if it satisfies the following conditions:

$$
\begin{aligned}
& \mathscr{D}_{X}(m) \mathscr{M}_{j} \subset \mathscr{M}_{m+j} \\
& \mathscr{D}_{X}(m) \mathscr{M}_{j}=\mathscr{M}_{m+j} \quad \text { for } \quad j \gg 0 \text { and } m \geqq 0 . \\
& \mathscr{M}_{j}=0 \quad \text { for } \quad j \ll 0 \\
& \mathscr{M}=\cup \mathscr{M}_{j} .
\end{aligned}
$$

For a coherent $\mathscr{D}_{X}$-Module $\mathscr{M}$, we donote by $\mathrm{Ch}(\mathscr{M})$ the support of $\mathcal{O}_{T^{*} X} \underset{\operatorname{gr}_{\mathscr{D}_{X}}}{\bigotimes} \operatorname{gr} \mathscr{M}$ where gr $\mathscr{M}$ denotes $\oplus \mathscr{M}_{j} / \mathscr{M}_{j-1}$ for a good filtration of $\mathscr{M}$. Since $\mathrm{Ch} \mathscr{M}$ does not depend on the choice of good filtration and since a coherent $\mathscr{D}_{X}$-Module has locally a good filtration, $\mathrm{Ch}(\mathscr{M})$ is a well-defined closed homogeneous analytic subvariety of $T^{*} X$. We call $\mathrm{Ch}(\mathscr{M})$ the characteristic variety of $\mathscr{M}$. It is shown that the characteristic variety is always involutive (Theorem 5.3.2 [SKK]), and in particular its dimension is equal to or larger than the dimension of $X$.

Definition 5. 1. If the characteristic variety of a coherent $\mathscr{D}_{X^{-}}$ Module $\mathscr{M}$ has the same dimension as $X, \mathscr{M}$ is called holonomic.

Definition 5. 2. Let $\mathscr{M}$ be a holonomic $\mathscr{D}_{X}$-Module. If the following condition is satisfied, $\mathscr{M}$ is called regular (in [KK], it is called with R. S.)

(5.1.5) $\mathscr{M}$ has a good filtration $\left\{\mathscr{M}_{j}\right\}$ such that $f \operatorname{gr} \mathscr{M}=0$ for any $f \in \operatorname{gr} \mathscr{D}_{X}$ vanishing on $\mathrm{Ch}(\mathscr{M})$.

Example 5. 1. $\mathcal{O}_{X}$ is a regular holonomic $\mathscr{D}_{X}$-Module and $\mathrm{Ch}\left(\mathcal{O}_{X}\right)$ coincides with the zero section of $T^{*} X$. 
Example 5. 2. $X \subset C$ and $\mathscr{M}=\mathscr{D}_{X} / \mathscr{D}_{X} P$ for $P \neq 0 \in \mathscr{D}_{X}$. In this case $\mathscr{M}$ is always holonomic, and $\mathscr{M}$ is regular if and only if $P u=0$ has regular singularities in the sense used in the theory of linear ordinary differential equations.

Example 5. 3. $X=\mathbb{C}^{n}, \quad A_{j} \in M_{N}(\boldsymbol{C}) \quad(j=1, \ldots, n)$. Then, $\mathscr{M}=$ $\mathscr{D}_{X}^{N} / \sum_{j=1}^{n} \mathscr{D}_{X}^{N}\left(x_{j} \frac{\partial}{\partial x_{j}}-A_{j}\right)$ is a regular holonomic $\mathscr{D}_{X}$-Module. If $A_{j}$ 's commute to each other, we have

$$
\operatorname{Ch}(\mathscr{M})=\left\{(x, \xi) \in T^{*} X ; x_{j} \xi_{j}=0 \quad j=1, \ldots, n\right\} .
$$

5. 2. Now, we shall give several properties of regular holonomic $\mathscr{D}$-Modules.

Proposition 5. 3. (1) A coherent sub-Module and a coherent quotient of a regular holonomic $\mathscr{D}$-Module are also regular holonomic. (2) If $\mathscr{M}_{1} \rightarrow \mathscr{M}_{2} \rightarrow \mathscr{M}_{3} \rightarrow \mathscr{M}_{4} \rightarrow \mathscr{M}_{5}$ is an exact sequence of $\mathscr{D}-$ Modules and if $\mathscr{M}_{1}, \mathscr{M}_{2}, \mathscr{M}_{4}$ and $\mathscr{M}_{5}$ are regular holonomic then so is $\mathscr{M}_{3}$.

(3) (Cor. 5.1.1. [KK]) The notion of regular holonomicity is local. (4) (Theorem 5.4.1. [KK]) Let $f$ be a holomorphic function and $\mathscr{M}$ a regular holonomic $\mathscr{D}$-Module. Then the localization $\mathscr{M}_{f}$ is also a regular holonomic $\mathscr{D}$-Module.

5. 3. Let $f: X \rightarrow Y$ be a holomorphic map from a complex manifold $X$ to a complex manifold $Y$. Set $\mathscr{D}_{X \rightarrow Y}=\mathscr{O}_{X} \otimes_{f^{-1} \mathscr{D}_{Y}} f^{-1} \mathscr{D}_{Y}$ and $\mathscr{D}_{Y \leftarrow X}=$ $f^{-1}\left(\mathscr{D}_{Y} \otimes_{\mathscr{D}_{Y}}^{\otimes} \Omega_{Y}^{\otimes-1}\right) \underset{f^{-1} \mathscr{D}_{Y}}{\otimes} \Omega_{X}$, where $\Omega_{Y}$ and $\Omega_{X}$ are the sheaves of the highestdegree holomorphic forms on $Y$ and $X$, respectively. Then $\mathscr{D}_{X \rightarrow Y}$ is a $\left(\mathscr{D}_{X}, f^{-1} \mathscr{D}_{Y}\right)$-bi-Module and $\mathscr{D}_{Y \leftarrow X}$ is a $\left(f^{-1} \mathscr{D}_{Y}, \mathscr{D}_{X}\right)$-bi-Module (see $[\mathrm{SKK}]$ ).

Proposition 5. 4. (Corollary 5.4.8 [KK]). If $\mathscr{N}$ is a regular holonomic $\mathscr{D}_{Y}-$ Module, then $\mathscr{F}_{0 x_{i}}^{\mathscr{D}_{Y}}\left(\mathscr{D}_{X \rightarrow Y}, \mathscr{N}\right)$ is a regular holonomic $\mathscr{D}_{\mathrm{X}}$-Module.

Proposition 5. 5. (Theorem 6.2.1 [KK]). If $\mathscr{K}$ is a regular holonomic $\mathscr{D}_{X}$-Module and if $f$ is projective, then $R^{i} f_{*}\left(\mathscr{D}_{Y \leftarrow X} \stackrel{L}{\underset{\mathscr{D}_{X}}{\otimes}} \mathscr{M}\right)$ is a regular holonomic $\mathscr{D}_{Y}$-Module. Here $\stackrel{\mathscr{D}}{X}_{X}^{L}$ means the left derived functor of $\underset{\mathscr{\mathscr { O }}_{X}}{\otimes}$ in the appropriate derived categories. 
5. 4. A sheaf of vector spaces $F$ on $X$ is called constructible if there exists a decreasing sequence of closed analytic subset $X_{0}=X \supset X_{1} \supset X_{2}$ $\supset \cdots$ of $X$ such that $\left.F\right|_{X_{j}-X_{j+1}}$ is locally constant of finite rank and $\cap X_{j}=\phi$.

Proposition 5.6 ([K1]). For a holonomic $\mathscr{D}_{X}$-Module $\mathscr{M}$, $\mathscr{E} x \epsilon_{\mathscr{D}}^{j_{X}}\left(\mathscr{M}, \mathcal{O}_{X}\right)$ is a constructible sheaf for any $j$.

5. 5. We denote by $\mathscr{D}_{X}^{\infty}$ the sheaf of linear differential operators of infinite order (see [SKK]). Then $\mathscr{D}_{X}^{\infty}$ is faithfully flat over $\mathscr{D}_{X}, \mathscr{O}_{X}$ is a left $\mathscr{D}_{X}^{\infty}$-Module and $\Omega_{X}$ is a right $\mathscr{D}_{X}^{\infty}$-Module. A $\mathscr{D}_{X}^{\infty}$-Module $\mathscr{M}$ is called holonomic if there exists a holonomic $\mathscr{D}_{X}$-Module $\mathscr{M}^{\prime}$ such that $\mathscr{M} \cong \mathscr{D}_{X}^{\infty} \otimes_{\mathscr{D}_{X}} \mathscr{M}^{\prime}$.

Proposition 5.7 (Theorem 5.2.1 [KK])。 Let $\mathscr{M}$ be a holonomic $\mathscr{D}_{X}^{\infty}$-Module and set $\mathscr{M}_{\text {reg }}=\left\{u \in \mathscr{M} ; \mathscr{D} u\right.$ is a regular holonomic $\mathscr{D}_{X^{-}}$ Module\}. Then $\mathscr{M}_{\text {reg }}$ is a regular holonomic $\mathscr{D}_{X}$-Module and we have

$$
\mathscr{U}=\mathscr{D}_{X}^{\infty} \otimes_{\mathscr{D}_{X}}^{\otimes} \mathscr{l}_{\text {reg }} .
$$

In particular for any holonomic $\mathscr{D}_{X}$-Module $\mathscr{l}$ (not necessarily regular), there exists a regular holonomic $\mathscr{D}_{X}$-Module $\mathscr{M}^{\prime}$ (unique up to an isomorphism) such that $\mathscr{D}_{X}^{\infty} \otimes_{\mathscr{D}_{X}} \mathscr{H}$ is isomorphic to $\mathscr{D}_{\mathscr{D}_{X}}^{\infty} \otimes_{\mathscr{M}^{\prime}} \mathscr{M}^{\prime}$.

\section{§6. Statement of the Main Theorem}

6. 1. Let $X$ be a paracompact complex manifold of dimension $n$, $X_{R}$ the underlying real analytic manifold and $\bar{X}$ the complex conjugate of $X$. Thus, by the diagonal map $X_{R} \rightarrow X \times \bar{X}, X \times \bar{X}$ is regarded as a complexification of $X_{R}$. The ring $\mathscr{A}_{X_{R}}$ (resp. $\mathscr{D}_{X_{R}}$ ) coincides with the restriction of $\mathscr{O}_{X \times \bar{X}}$ (resp. $\mathscr{D}_{X \times \bar{X}}$ ). By this, we regard $\mathscr{D}_{X}$ and $\mathscr{D}_{\bar{X}}$ as a sub-Ring of $\mathscr{D}_{X_{R}}$. For a $\mathscr{D}_{X_{R}}$-Module $\mathscr{H}, \mathbb{R} \mathscr{H}_{m_{\mathscr{D}_{\bar{X}}}}\left(\mathscr{O}_{\bar{X}}, \mathscr{M}\right)$ is quasi-isomorphic to the Dolbeaut complex with $\mathscr{I}$ as coefficients:

$$
\mathscr{M} \stackrel{\bar{\partial}}{\longrightarrow} \Omega_{X_{R}}^{(0,1)} \bigotimes_{\mathscr{A}} \mathscr{H} \stackrel{\bar{\partial}}{\longrightarrow} \Omega_{X_{R}}^{(0,2)} \bigotimes_{\mathscr{A}} \mathscr{M l} \longrightarrow \cdots \stackrel{\bar{\partial}}{\longrightarrow} \Omega_{X_{R}}^{(0, n)} \bigotimes_{\mathscr{A}} \mathscr{M} .
$$

Here $\Omega_{X_{\boldsymbol{R}}}^{(\phi, q)}$ is the sheaf of $(p, q)$-forms, with real analytic function as coefficients. 
6. 2. Let $\operatorname{Mod}(X)$ denote the category of sheaves of $\boldsymbol{C}$-vector spaces on $X$. Let $\mathrm{D}(X)$ denote its derived category and let $\mathrm{D}_{\mathrm{c}}^{b}(X)$ denote the full sub-category of $\mathrm{D}(X)$ consisting of bounded complexes $F^{\text {. }}$ such that $\mathscr{H}^{j}\left(F^{*}\right)$ is constructible for any $j$.

Let $\operatorname{Mod}\left(\mathscr{D}_{X}\right)$ denote the category of $\mathscr{D}_{X}$-Modules and $\mathrm{D}\left(\mathscr{D}_{X}\right)$ its derived category. We denote by $\mathrm{D}_{\mathrm{rh}}^{\mathrm{b}}\left(\mathscr{D}_{X}\right)$ the full subcategory of $\mathrm{D}\left(\mathscr{D}_{X}\right)$ consisting of bounded complexes $\mathscr{M}^{\circ}$ such that $\mathscr{H}^{j}\left(\mathscr{M}^{*}\right)$ is a regular holonomic $\mathscr{D}_{X}$-Module for any $j$.

By replacing $\mathscr{D}_{X}$ with $\mathscr{D}_{X}^{\infty}$ and regular holonomic with holonomic $\mathscr{D}_{X}^{\infty}$-Module, we similarly define $\operatorname{Mod}\left(\mathscr{D}_{X}^{\infty}\right), \mathrm{D}\left(\mathscr{D}_{X}^{\infty}\right)$ and $\mathrm{D}_{\mathrm{h}}^{\mathrm{b}}\left(\mathscr{D}_{X}^{\infty}\right)$.

6. 3. We shall define functors

$$
\begin{aligned}
& J_{X}: \mathrm{D}_{\mathrm{rh}}^{\mathrm{b}}\left(\mathscr{D}_{X}\right) \rightarrow \mathrm{D}_{\mathrm{h}}^{\mathrm{b}}\left(\mathscr{D}_{X}^{\infty}\right), \\
& \Phi_{X}: \mathrm{D}_{\mathrm{rh}}^{\mathrm{b}}\left(\mathscr{D}_{X}\right) \rightarrow \mathrm{D}_{\mathrm{c}}^{\mathrm{b}}(X)^{\circ}, \\
& \Phi_{X}^{\infty}: \mathrm{D}_{\mathrm{h}}^{\mathrm{b}}\left(\mathscr{D}_{X}^{\infty}\right) \rightarrow \mathrm{D}_{\mathrm{c}}^{\mathrm{b}}(X)^{\circ}, \\
& \Psi_{X}: \mathrm{D}_{\mathrm{c}}^{\mathrm{b}}(X)^{\circ} \rightarrow \mathrm{D}\left(\mathscr{D}_{X}\right), \text { and } \\
& \Psi_{X}^{\infty}: \mathrm{D}_{\mathrm{c}}^{\mathrm{b}}(X)^{\circ} \rightarrow \mathrm{D}\left(\mathscr{D}_{X}^{\infty}\right)
\end{aligned}
$$

as follows

$$
\begin{aligned}
& J_{X}=\mathscr{D}_{X}^{\infty} \mathscr{D}_{X}^{\bigotimes} \\
& \Phi_{X}^{\infty}=\boldsymbol{R} \mathscr{H}^{\circ} m_{\mathscr{D}_{X}^{\infty}}\left(*, \mathcal{O}_{X}\right) \\
& \Phi_{X}=\Phi_{X}^{\infty} \circ J=\boldsymbol{R} \mathscr{H}^{\circ} m_{\mathscr{D}_{X}}\left(*, \mathcal{O}_{X}\right) \\
& \Psi_{X}^{\infty}=\boldsymbol{R} \mathscr{H o m}_{C_{X}}\left(*, \mathcal{O}_{X}\right) \text {. }
\end{aligned}
$$

Here ${ }^{\circ}$ denotes the opposite category. Since $\mathscr{D}_{X}^{\infty}$ is faithfully flat over $\mathscr{D}_{X}, J_{X}$ is well-defined. By Proposition 5.6, $\Phi_{X}$ and $\Phi_{X}^{\infty}$ are well-defined.

Now, we shall define the functor $\Psi_{X}$. This is obtained as the composition of $\mathrm{D}_{\mathrm{c}}^{\mathrm{b}}(X)^{\circ} \rightarrow \mathrm{D}_{\boldsymbol{R}-\mathrm{c}}^{\mathrm{b}}\left(X_{\boldsymbol{R}}\right)^{\circ} \stackrel{\boldsymbol{R} T H}{\longrightarrow} \mathrm{D}^{\mathrm{b}}\left(\mathscr{D}_{X_{\boldsymbol{R}}}\right)$ and $\boldsymbol{R} \mathscr{H}_{0} m_{\mathscr{D}_{\bar{X}}}\left(\mathcal{O}_{\bar{X}}, *\right)$ : $\mathrm{D}^{\mathrm{b}}\left(\mathscr{D}_{X_{R}}\right) \rightarrow \mathrm{D}\left(\mathscr{D}_{X}\right)$.

Here $\mathrm{D}\left(\mathscr{D}_{X_{R}}\right)$ is the derived category of the abelian category of $\mathscr{D}_{X_{R}}$-Modules. Hence for $F^{*} \in \mathrm{Ob}\left(\mathrm{D}_{\mathrm{c}}^{\mathrm{b}}(X)\right), \Psi\left(F^{*}\right)$ is the Dolbeaut complex

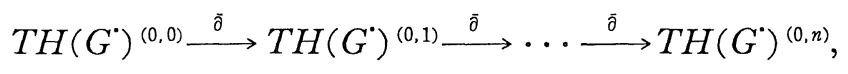

where $G^{*}$ is a complex of $\boldsymbol{R}$-constructible sheaves on $X_{R}$, which is quasi-isomorphic to $F^{*}$. We sometimes denote by the same letter $\Psi_{X}$ the functor $\boldsymbol{R} \mathscr{H}_{0} m_{\mathscr{D}_{\bar{X}}}\left(\mathcal{O}_{\bar{X}}, \boldsymbol{R} T H(*)\right)$ from $\mathrm{D}_{R-\mathrm{c}}^{\mathrm{b}}\left(X_{R}\right)^{\circ}$ to $\mathrm{D}\left(\mathscr{D}_{X}\right)$. 
In the sequel, we omit the subscript $X$ if there is no afraid of confusion.

Main Theorem. ( 1$) \Psi\left(\mathrm{D}_{\mathrm{c}}^{\mathrm{b}}(X)^{\circ}\right) \subset \mathrm{D}_{\mathrm{rh}}^{\mathrm{b}}\left(\mathscr{D}_{X}\right)$ and $\Psi^{\circ}=J_{\circ} \Psi$. (2) $J, \Phi, \Phi^{\infty}, \Psi$ and $\Psi^{\infty}$ give the equivalence between the categories $\mathrm{D}_{\mathrm{c}}^{\mathrm{b}}(X)^{\circ}, \mathrm{D}_{\mathrm{rh}}^{\mathrm{b}}\left(\mathscr{D}_{X}\right)$ and $\mathrm{D}_{\mathrm{h}}^{\mathrm{b}}\left(\mathscr{D}_{X}^{\infty}\right)$. Moreover, $\Phi$ and $\Psi\left(\right.$ resp. $\Phi^{\infty}$ and $\left.\Psi^{\infty}\right)$ are inverse to each other.

In $[\mathrm{KK}]$, we proved the following

Lemma 6. 1. ( 1 ) (Proposition 1.4.8., Theorem 6.1.1 [KK]) $J, \Phi$ and $\Phi^{\infty}$ are faithful.

(2) (Theorem 1.4.9[KK]) $\Psi^{\infty} \circ \Phi^{\infty}=\mathrm{id}$.

Hence Main Theorem is a consequence of the following two propositions.

Proposition 6. 2. $\Psi\left(\mathrm{D}_{\mathrm{c}}^{\mathrm{b}}(X)^{\circ}\right) \subset \mathrm{D}_{\mathrm{rh}}^{\mathrm{b}}\left(\mathscr{D}_{X}\right)$ 。

Proposition 6. 3. $\Phi \Psi=\mathrm{id}$.

However, the latter follows easily from the first. In order to show this, let $F^{*}$ be an object of $\mathrm{D}_{c}^{\mathrm{b}}(X)$. We have

$$
\begin{aligned}
\Phi \Psi\left(F^{*}\right) & =\boldsymbol{R} \mathscr{H o m}_{\mathscr{D}_{X}}\left(\Psi\left(F^{*}\right), \mathcal{O}_{X}\right) \\
& =\boldsymbol{R} \mathscr{H}_{0 m_{C_{X}}}\left(\boldsymbol{R} \mathscr{H} o m_{\mathscr{D}_{X}}\left(\mathcal{O}_{X}, \Psi\left(F^{*}\right)\right), \boldsymbol{C}_{X}\right) .
\end{aligned}
$$

Here we used Proposition 1.4.6. $[\mathrm{KK}]$ and the fact that $\Psi\left(F^{*}\right) \in$ $\mathrm{Ob}\left(\mathrm{D}_{\mathrm{rh}}^{\mathrm{b}}\left(\mathscr{D}_{X}\right)\right)$. On the other hand, we have

$$
\begin{aligned}
& \boldsymbol{R} \mathscr{H} o m_{\mathscr{D}_{X}}\left(\mathcal{O}_{X}, \Psi\left(F^{*}\right)\right)=\boldsymbol{R} \mathscr{H o m}_{\mathscr{D}_{X}}\left(\mathcal{O}_{X}, \boldsymbol{R} \mathscr{H}_{0 m_{\mathscr{D}_{\bar{X}}}}\left(\mathcal{O}_{\bar{X}}, \boldsymbol{R} T H\left(F^{*}\right)\right)\right) \\
& =\boldsymbol{R} \mathscr{H} \circ m_{\mathscr{D}_{X}}\left(\mathscr{A}_{X_{\boldsymbol{R}}}, \boldsymbol{R} T H\left(F^{*}\right)\right),
\end{aligned}
$$

which equals $\boldsymbol{R} \mathscr{H} o m\left(F^{*}, \boldsymbol{C}_{X}\right)$ by Proposition 4. 6. Thus Proposition 6. 3 follows from Proposition 6. 2.

\section{§7. Proof of Main Theorem}

7. 1. We have already reduced Main Theorem to Proposition 6. 2. We shall prove this by using Hironaka's desingularization theorem. In order to perform this, we have to know how the functor $\Psi$ behaves under integration. 
7. 2 Let $X$ and $Y$ be two complex manifolds and $f: X \rightarrow Y$ a holomorphic map.

Proposition 7. 1. Suppose that an object $F^{*}$ of $\mathrm{D}_{R-\mathrm{c}}^{\mathrm{b}}\left(X_{R}\right)$ of finite rank satisfies the following condition:

(7.2.1) The closure of $\operatorname{Supp} \mathscr{H}^{j}\left(F^{*}\right)$ is proper over $Y$ for any $j$. Then we have

$$
\begin{gathered}
\boldsymbol{R} f_{*}\left(\mathscr{D}_{Y_{\leftarrow} X} \bigotimes_{\mathscr{D}_{X}}^{L} \Psi_{X}\left(F^{*}\right)\right)[\operatorname{dim} X] \\
\cong \Psi_{Y}\left(\boldsymbol{R} f_{*}\left(F^{*}\right)\right)[\operatorname{dim} Y] .
\end{gathered}
$$

Proof. By Theorem 4. 1, we have

$$
\begin{aligned}
& \boldsymbol{R} f_{*}\left(\mathscr{D}_{Y_{\boldsymbol{R}^{\leftarrow} X_{\boldsymbol{R}}}} \bigotimes_{\mathscr{D}_{X_{\boldsymbol{R}}}}^{\boldsymbol{R}} \boldsymbol{R} T H_{X_{\boldsymbol{R}}}\left(F^{*}\right)\right) \\
& \cong \boldsymbol{R} T H_{Y_{\boldsymbol{R}}}\left(\boldsymbol{R} f_{*}\left(F^{*}\right)\right) .
\end{aligned}
$$

Hence we have

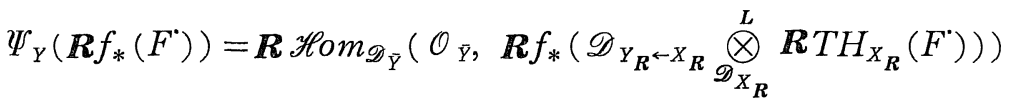

$$
\begin{aligned}
& =\boldsymbol{R} f_{*}\left(\boldsymbol{R} \mathscr{H}_{0 m_{\mathscr{D}_{\hat{Y}}}}\left(\mathcal{O}_{\bar{Y}}, \mathscr{D}_{Y_{\boldsymbol{R}} \leftarrow X_{\boldsymbol{R}}} \underset{\mathscr{D}_{X} \boldsymbol{R}}{\boldsymbol{L}} \boldsymbol{R} T H_{X_{\boldsymbol{R}}}\left(F^{*}\right)\right)\right) \text {. }
\end{aligned}
$$

Thus the proposition is a consequence of the following lemma.

Lemma 7. 2. $\quad \boldsymbol{R} \mathscr{H}_{0} m_{\mathscr{D}_{\bar{Y}}}\left(\mathcal{O}_{\bar{Y}}, \mathscr{D}_{Y_{\boldsymbol{R}} \leftarrow X_{\boldsymbol{R}}}\right)$

$$
=\mathscr{D}_{Y \leftarrow-X}{\underset{\mathscr{D}}{X}}_{X}^{L} \boldsymbol{R} \mathscr{H}_{0} m_{\mathscr{D}_{\bar{X}}}\left(\mathcal{O}_{\bar{X}}, \mathscr{D}_{X_{\boldsymbol{R}}}\right)[\operatorname{dim} X-\operatorname{dim} Y] .
$$

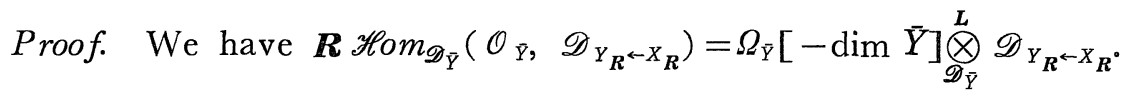

We have also

$$
\mathscr{D}_{Y_{\boldsymbol{R}} \leftarrow X}=\left.\mathscr{D}_{Y \times \bar{Y} \leftarrow X \times \bar{X}}\right|_{X_{\boldsymbol{R}}}
$$

and in general

$$
\mathscr{D}_{U \leftarrow-V} \bigotimes_{\mathscr{D}_{V}}^{L} \mathscr{D}_{V \times W}=\mathscr{D}_{U \times W \leftarrow V \times W} .
$$

These imply

$$
\begin{aligned}
\boldsymbol{R} \mathscr{H} \operatorname{m}_{\mathscr{D}_{\bar{Y}}}\left(\mathcal{O}_{\bar{Y}}, \mathscr{D}_{Y_{\boldsymbol{R}} \leftarrow X_{\boldsymbol{R}}}\right) \\
\quad=\left.\mathscr{D}_{p t \leftarrow \bar{Y}}{\underset{\mathscr{D}}{\mathscr{Y}}}^{L} \mathscr{D}_{Y \times \bar{Y} \leftarrow X \times \bar{X}}\right|_{X_{\boldsymbol{R}}}[-\operatorname{dim} Y]
\end{aligned}
$$




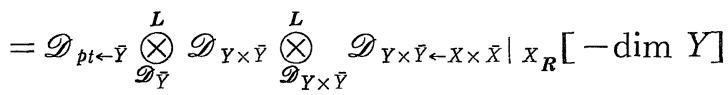

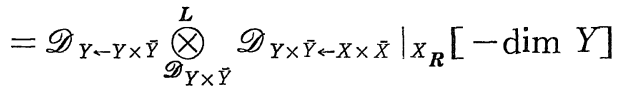

$$
\begin{aligned}
& =\left.\mathscr{D}_{Y \leftarrow X \times \bar{X}}\right|_{X_{R}}[-\operatorname{dim} Y] \text {. }
\end{aligned}
$$

On the other hand, we have

$$
\begin{aligned}
& \mathscr{D}_{Y \leftarrow X}{\stackrel{\bigotimes}{\mathscr{D}_{X}}}_{\mid L} \boldsymbol{R} \mathscr{H}_{0} m_{\mathscr{D}_{\bar{X}}}\left(\mathcal{O}_{\bar{X}}, \mathscr{D}_{X_{R}}\right)
\end{aligned}
$$

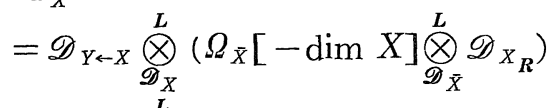

$$
\begin{aligned}
& =\left.\mathscr{D}_{Y \leftarrow X} \stackrel{\mathscr{D}}{X}_{X}^{L} \mathscr{D}_{X \leftarrow X \times \bar{X}}\right|_{X_{R}}[-\operatorname{dim} X] \\
& =\left.\mathscr{D}_{Y \leftarrow X \times \bar{X}}\right|_{X_{R}}[-\operatorname{dim} X] \text {. }
\end{aligned}
$$

The following lemma follows immediately from Proposition 5. 3.

Lemma 7. 3. If $\underset{F^{\prime} \rightarrow F^{\prime \prime}}{F^{\prime}}$ is a distinguished triangle in $\mathrm{D}_{\mathrm{c}}^{\mathrm{b}}(X)$ and if two of $\Psi_{X}\left(F^{\prime}\right), \Psi_{X}\left(F^{*}\right)$ and $\Psi_{X}\left(F^{\prime \prime}\right)$ belong to $\mathrm{D}_{\mathrm{rh}}^{\mathrm{b}}\left(\mathscr{D}_{X}\right)$, then so does the other.

7. 3. Now, we shall prove Proposition 6. 2. By the standard argument using Lemma 7.3, it is enough to show $\Psi_{X}(F) \in \mathrm{Ob}\left(\mathrm{D}_{\mathrm{rh}}^{\mathrm{b}}\left(\mathscr{D}_{X}\right)\right)$ for any constructible sheaf $F$. We shall prove this by the induction of the dimension of the closure $Z$ of the support of $F$.

Let $Z_{0}$ be a nowhere dense closed analytic subset of $Z$ such that

$$
\left.F\right|_{Z-Z_{0}} \text { is locally constant. }
$$

$Z-Z_{0}$ is non-singular.

By the hypothesis of the induction, $\Psi_{X}\left(F_{Z_{0}}\right)$ belongs to $\mathrm{D}_{\mathrm{rh}}^{\mathrm{b}}\left(\mathscr{D}_{X}\right)$. Hence by using Lemma 7. 3, we may assume $F_{Z_{0}}=0$ without loss of generality. Let $f: X^{\prime} \rightarrow X$ be a projective map satisfying the following conditions

(7.3.3) $X^{\prime}$ is non-singular and $f\left(X^{\prime}\right)=Z$.

(7. 3.4) $Z_{0}^{\prime}=f^{-1}\left(Z_{0}\right)$ is a normally crossing hypersurface of $X^{\prime}$.

(7.3.5) $\quad X^{\prime}-Z_{0}^{\prime} \rightarrow Z-Z_{0}$ is an isomorphism.

Such an $f$ is obtained by a succession of blowing up's by Hironaka's desingularization theorem [H1]. 
Set $F^{\prime}=f^{-1}(F)$. Then we have

$$
\left.F^{\prime}\right|_{z_{0}^{\prime}}=0
$$

$$
\left.F^{\prime}\right|_{X^{\prime}-z_{0}^{\prime}} \text { is locally constant, }
$$

As we shall prove later in Lemma 7. 4, $\Psi_{X^{\prime}}\left(F^{\prime}\right)$ belongs to $\mathrm{Ob}\left(\mathrm{D}_{\mathrm{rh}}^{\mathrm{b}}\right.$ $\left.\left(\mathscr{D}_{X^{\prime}}\right)\right)$. By Proposition 7. 1 and Proposition 5. 5 and (7.3.8), $\Psi_{X}(F)$ belongs to $\mathrm{D}_{\mathrm{rh}}^{\mathrm{b}}\left(\mathscr{D}_{X}\right)$.

7. 4. So far, we reduced Main Theorem to the following lemma.

Lemma 7. 4. Let $Y$ be a normally crossing hypersurface of a complex manifold $X$, and $F$ a constructible sheaf on $X$ such that $\left.F\right|_{Y}=0$ and that $\left.F\right|_{X-Y}$ is locally constant of finite rank. Then $\Psi_{X}(F)$ belongs to $\mathrm{D}_{\mathrm{rh}}^{\mathrm{b}}\left(\mathscr{D}_{X}\right)$.

We shall prove first the following special case.

Lemma 7. 5. Being $X$ and $Y$ in the preceding lemma, $\Psi_{X}\left(\boldsymbol{C}_{X-Y}\right)$ is isomorphic to the sheaf $\mathscr{H}_{[X \mid Y]}^{0}\left(\mathcal{O}_{X}\right)$ of meromorphic functions on $X$ with possible poles in $Y$.

Proof. If $Y=\emptyset$, then $\Psi_{X}\left(\boldsymbol{C}_{X}\right)$ is nothing but the Dolbeaut complex

$$
0 \longrightarrow \mathscr{D} \mathfrak{k}^{(0,0)} \stackrel{\bar{\partial}}{\longrightarrow} \mathscr{D} \mathfrak{k}^{(0,1)} \stackrel{\bar{\partial}}{\longrightarrow} \cdots \longrightarrow \mathscr{D} \mathfrak{k}^{(0, n)} \longrightarrow 0 .
$$

Therefore, $\Psi_{X}\left(\boldsymbol{C}_{X}\right)$ is isomorphic to $\mathcal{O}_{X}$. The question being local, we may assume

$$
X=C^{n}, \text { and } Y=\bigcup_{j=1}^{l} Y_{j}
$$

where $Y_{j}=\left\{x=\left(x_{1}, \ldots, x_{n}\right) \in X ; x_{j}=0\right\}$.

Set $f=x_{1} \cdots x_{l}$. Then, by Proposition 3.23 we have

$$
T H\left(\boldsymbol{C}_{X-Y}\right)=T H\left(\boldsymbol{C}_{X}\right)_{f}=\left(\mathscr{D} k_{X_{R}}\right)_{f} .
$$

Here, $*_{f}$ means the localization by $f$. On the other hand, we have

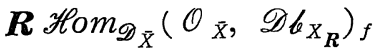

$$
\begin{aligned}
& =\left(\mathcal{O}_{X}\right)_{f}=\mathscr{H}_{[X \mid Y]}^{0}\left(\mathcal{O}_{X}\right) \text {. Q. E. D. }
\end{aligned}
$$


Now, we shall prove Lemma 7.4. The question being local, we may assume (7.4.1) with $l \geqq 1$. Thus $F$ is determined by the monodromy around $Y_{j}$. Let $\exp 2 \pi \sqrt{-1} A_{j}$ be the monodromy around $Y_{j}$, with an $(N \times N)$-matrix $A_{j}$. We may assume

$$
\left[A_{j}, A_{k}\right]=0 \text { for } 1 \leqq j, k \leqq l .
$$

Then, by multiplying $x^{A}=x_{1}^{A_{1}} x_{2}^{A_{2}} \cdots x_{l}^{A_{l}}, T H(F)$ is isomorphic to $T H\left(\boldsymbol{C}_{X-Y}^{N}\right)$. This is not $\mathscr{D}_{X}$-linear but $\mathscr{D}_{\bar{X}}$-linear, and $\mathcal{O}_{X}$-linear. Hence as an $\mathcal{O}_{X}$-Module, $T H(F)$ is isomorphic to $T H\left(\boldsymbol{C}_{X-Y}^{N}\right)=$ $\mathscr{H}_{[X \mid Y]}^{0}\left(\mathcal{O}_{X}\right)^{N}$. If we denote by $f$ this isomorphism, the action of $\partial / \partial x_{j}$ is given

$$
f\left(\partial / \partial x_{j} \cdot u\right)=\left(\partial / \partial x_{j}-A_{j} x_{j}^{-1}\right) f(u) \quad \text { for } \quad u \in T H(F) .
$$

Here $A_{j}(j>l)$ is understood to be zero. One can easily show that $\mathscr{H}_{[X \mid Y]}^{0}\left(\mathcal{O}_{X}\right)^{N}$ with this structure of $\mathscr{D}_{X}$-Module is regular holonomic. This shows Lemma 7.4 and hence the proof of Main Theorem is completed.

\section{§8. Applications}

8. 1. In $[K K]$, we have proved that the integration of regular holonomic $\mathscr{D}$-Module under projective morphism is regular holonomic. This can be generalized to proper morphisms.

Theorem 8. 1. Let $f: X \rightarrow Y$ be a morphism and let dll be a regular holonomic $\mathscr{D}_{X}$-Module whose support is proper over $Y$. Then any cohomology of $\int_{f} \mathscr{M}=\boldsymbol{R} f_{*}\left(\mathscr{D}_{Y \leftarrow X} \underset{\mathscr{D}_{X}}{\otimes} \mathscr{M}\right)$ is regular holonomic.

Proof. Set $F^{*}=\Psi_{X}(\mathscr{M})$. Then by Proposition 7. 1,

$$
\int_{f} \mathscr{M} \cong \Psi_{Y}\left(\boldsymbol{R} f_{*}\left(F^{*}\right)\right)[\operatorname{dim} Y-\operatorname{dim} X] .
$$

Since $\boldsymbol{R} f_{*}\left(F^{*}\right)$ belongs to $\mathrm{D}_{\mathrm{c}}^{\mathrm{b}}(Y), \int_{f} \mathscr{M}$ belongs to $\mathrm{D}_{\mathrm{rh}}^{\mathrm{b}}\left(\mathscr{D}_{Y}\right)$.

Q. E.D.

8. 2. Let $M$ be a real analytic manifold and $X$ a complexification of $M$. Then we have, for any $F^{*} \in \mathrm{D}_{R-\mathrm{c}}^{\mathrm{b}}(M)$,

$$
\boldsymbol{R} T H\left(F^{*}\right) \rightarrow \boldsymbol{R} \mathscr{H o m}_{C}\left(F^{*}, \mathscr{B}_{M}\right) \text {. }
$$


Theorem 8. 2. For any $\mathscr{M}^{\prime} \in \mathrm{D}_{\mathrm{rh}}^{\mathrm{b}}\left(\mathscr{D}_{X}\right)$ and for any $F^{*} \in \mathrm{D}_{R-\mathrm{c}}^{\mathrm{b}}(M)$ of finite rank,

$$
\begin{aligned}
& \boldsymbol{R} \mathscr{H} m_{\mathscr{D}_{X}}\left(\mathscr{M} ; \boldsymbol{R} T H\left(F^{*}\right)\right) \\
& \rightarrow \boldsymbol{R} \mathscr{H} m_{\mathscr{D}_{X}}\left(\mathscr{M}^{*}, \boldsymbol{R} \mathscr{H} m_{C}\left(F^{*}, \mathscr{B}_{M}\right)\right)
\end{aligned}
$$

is an isomorphism.

In particular, if we set $F^{*}=\boldsymbol{C}_{M}$, we obtain

Corollary 8. 3. For any $\mathscr{M}^{*} \in \mathrm{D}_{\mathrm{rh}}^{\mathrm{b}}\left(\mathscr{D}_{X}\right)$

$$
\boldsymbol{R} \mathscr{H} 0 m_{\mathscr{D}_{X}}\left(\mathscr{M}, \mathscr{D} b_{M}\right) \rightarrow \boldsymbol{R} \mathscr{H} m_{\mathscr{D}_{X}}\left(\mathscr{M}, \mathscr{B}_{M}\right)
$$

is an isomorphism.

Note that $\boldsymbol{R} \mathscr{H} o m_{\mathscr{D}_{X}}\left(\mathscr{M}, \boldsymbol{R} \mathscr{H} o m_{C}\left(F, \mathscr{B}_{M}\right)\right)$ can be calculated as follows.

Since $\mathscr{B}_{M}=\boldsymbol{R} \Gamma_{M}\left(\mathcal{O}_{X}\right) \otimes \omega_{M}[\operatorname{dim} M]\left(\boldsymbol{\omega}_{M}=\mathscr{H}_{M}^{n}\left(\boldsymbol{C}_{X}\right)\right)$, we have

$$
\begin{aligned}
& \boldsymbol{R} \mathscr{H o m}_{\mathscr{D}_{X}}\left(\mathscr{M}, \boldsymbol{R} \mathscr{H}_{0} m_{C}\left(F, \mathscr{B}_{M}\right)\right) \\
& =\boldsymbol{R} \mathscr{H} o m_{c_{M}}\left(F, \boldsymbol{R} \mathscr{H}_{0} m_{\mathscr{D}_{X}}\left(\mathscr{M}^{\prime}, \mathscr{B}_{M}\right)\right) \\
& =\boldsymbol{R} \mathscr{H} o m_{c_{M}}\left(F, \boldsymbol{R} \Gamma_{M}\left(\boldsymbol{R} \mathscr{H} o m_{\mathscr{D}_{X}}\left(\mathscr{M} ; \mathcal{O}_{X}\right)\right) \otimes \omega_{M}[\operatorname{dim} M]\right) \\
& =\boldsymbol{R} \mathscr{H} o m_{c_{X}}\left(F^{*}, \Phi_{X}\left(\mathscr{M}^{\prime}\right)\right) \otimes \omega_{M}[\operatorname{dim} M] \\
& =\boldsymbol{R} \mathscr{H}_{0 m_{C_{X}}}\left(F^{*} \otimes_{C_{X}} D R_{X}\left(\mathscr{M}^{\prime}\right), \boldsymbol{C}_{X}\right) \otimes \omega_{M}[\operatorname{dim} M] \\
& =\boldsymbol{R} \mathscr{H} \mathrm{m}_{c_{M}}\left(\left.F^{*} \otimes D R_{X}\left(\mathscr{M}^{\circ}\right)\right|_{M}, \boldsymbol{R} \Gamma_{M}\left(\boldsymbol{C}_{X}\right)\right) \otimes \omega_{M}[\operatorname{dim} M] \\
& =\boldsymbol{R} \mathscr{H o m}_{C_{M}}\left(\left.F^{*}{\underset{C_{M}}{\otimes}}_{M} D R_{X}\left(\mathscr{M}^{\circ}\right)\right|_{M}, \boldsymbol{C}_{M}\right) .
\end{aligned}
$$

Here $D R_{X}\left(\mathscr{M}^{\prime}\right)=\boldsymbol{R} \mathscr{H}_{0 m_{\mathscr{D}_{X}}}\left(\mathcal{O}_{X}, \mathscr{M}^{\prime}\right)=\boldsymbol{R} \mathscr{H}_{0} m_{c_{X}}\left(\Phi_{X}\left(\mathscr{M}^{\prime}\right), \boldsymbol{C}_{X}\right)$.

Thus, we obtain

Corollary 8. 4. For any $\mathscr{M}^{*} \in \mathrm{D}_{\mathrm{rh}}^{\mathrm{b}}\left(\mathscr{D}_{X}\right)$ and $F^{*} \in \mathrm{D}_{R-\mathrm{c}}^{\mathrm{b}}(M)$, we have

$$
\begin{aligned}
& \boldsymbol{R} \mathscr{H o m}_{\mathscr{D}_{X}}\left(\mathscr{M}^{*}, \boldsymbol{R} T H\left(F^{*}\right)\right) \\
& \quad \cong \boldsymbol{R} \mathscr{H o m}_{c_{M}}\left(\left.F^{*} \otimes_{c_{M}} D R\left(\mathscr{M}^{\prime}\right)\right|_{M}, \boldsymbol{C}_{M}\right) .
\end{aligned}
$$

Corollary 8.5. For $\mathscr{M}^{*} \in \mathrm{D}_{\mathrm{rh}}^{\mathrm{b}}\left(\mathscr{D}_{X}\right)$

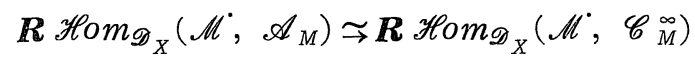

where $\mathscr{C}_{M}^{\infty}$ is the sheaf of $C^{\infty}$-functions on $M$.

Proof. We may assume $\mathscr{M}^{\circ}$ is a single complex. Let 


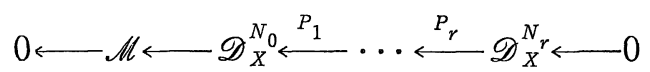

be a free resolution of $\mathscr{H}$. Then for any relatively compact open subanalytic set $U$

$$
\mathbb{R} \Gamma\left(U ; \boldsymbol{R} \mathscr{H} m_{\mathscr{D}_{X}}\left(\mathscr{M}, \mathscr{A}_{M}\right)\right)=\mathscr{A}_{M}(U) \cdot
$$

and

$$
\boldsymbol{R} \Gamma\left(U ; \boldsymbol{R} \mathscr{H}_{o m_{\mathscr{D}_{X}}}\left(\mathscr{M}, \mathscr{C}_{M}^{\infty}\right)\right)=\mathscr{C}_{M}^{\infty}(U)
$$

On the other hand, since the cohomology groups of $\Gamma_{c}\left(U, \mathscr{D}^{N^{N}}\right)$ $\stackrel{P_{r}^{*}}{\longleftarrow} \cdots \stackrel{P_{r}^{*}}{\longleftarrow} \Gamma_{c}\left(U, \mathscr{D} b^{N 0}\right)$ are finite dimensional, by the duality,

$$
\begin{aligned}
& \operatorname{Ext}_{\mathscr{D}}^{j}\left(U ; \mathscr{M}, \mathscr{C}_{M}^{\infty}\right) \\
& \quad=H_{c}^{n-j}\left(U ; \boldsymbol{R} \mathscr{H}_{o m_{\mathscr{D}}}\left(\mathscr{U}^{*}, \mathscr{D} b_{M}\right)\right)^{*}
\end{aligned}
$$

and similarly

$$
\text { (8. 2.4) } \operatorname{Ext}_{\mathscr{D}}^{j}\left(U ; \mathscr{M}, \mathscr{A}_{M}\right)=H_{c}^{n-j}\left(U ; \mathbb{R} \mathscr{H}_{0} m_{\mathscr{D}}\left(\mathscr{M}^{*}, \mathscr{B}_{M}\right)\right)^{*} \text {. }
$$

Thus Corollary 8.3 implies

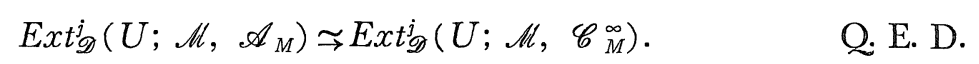

Corollary 8. 6. Let $X$ be a complex manifold. Then for any $F^{*} \in \mathrm{D}_{\boldsymbol{R}-\mathrm{c}}^{\mathrm{b}}\left(X_{R}\right)$ of finite rank and $\mathscr{M}^{\cdot} \in \mathrm{D}_{\mathrm{rh}}^{\mathrm{b}}\left(\mathscr{D}_{X}\right)$ we have

$$
\begin{aligned}
& \boldsymbol{R} \mathscr{H o m}_{\mathscr{D}_{X}}\left(\mathscr{M}^{*}, \Psi_{X}\left(F^{*}\right)\right) \\
& =\boldsymbol{R} \mathscr{H}_{0} m_{\mathscr{D}_{X}}\left(\mathscr{M}^{*}, \mathbb{R} \mathscr{H}_{0} m_{C_{X}}\left(F^{*}, \mathcal{O}_{X}\right)\right) \\
& =\boldsymbol{R} \mathscr{H}_{0} m_{C_{X}}\left(F^{*}, \Phi_{X}\left(\mathscr{M}^{*}\right)\right) \text {. }
\end{aligned}
$$

Proof. We have

$$
\begin{aligned}
& \mathbb{R} \mathscr{H o m}_{\mathscr{D}_{X}}\left(\mathscr{M}^{*}, \Psi_{X}\left(F^{*}\right)\right)
\end{aligned}
$$

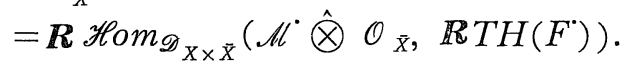

Since $\mathscr{M}^{\cdot} \hat{\otimes} \mathcal{O}_{\bar{X}} \in \mathrm{D}_{\mathrm{rh}}^{\mathrm{b}}\left(\mathscr{D}_{X \times \bar{X}}\right)$, the corollary follows from Theorem 8. 1 .

8. 3. We shall prove Theorem 8.2 first in the case where $F=\mathbb{C}_{M}$ by the induction on $\operatorname{Supp} \mathscr{H}^{\circ}=\bigcup_{j} \operatorname{Supp} \mathscr{H}^{j}\left(\mathscr{M}^{\circ}\right)$ and $\operatorname{dim} X$.

Let $Y$ be the union of the support of $\mathscr{M}^{\circ}$ and its complex conjugate. Then by the desingularization of Hironaka, there exist locally a real analytic manifold $M^{\prime}$, a complexification $X^{\prime}$ of $M^{\prime}$, a holomorphic map $f: X^{\prime} \rightarrow X$ and a nowhere dense analytic subset $Z$ of $Y$ satisfying the following conditions. 
(0) $Z$ is an intersection of $Y$ and a hypersurface of $X$.

(1) $f: X^{\prime} \rightarrow X$ is proper.

(2) $f\left(M^{\prime}\right) \subset M$.

(3) $f: X^{\prime}-f(Z) \rightarrow X-Z$ is an isomorphism.

(4) $Y^{\prime}=\overline{f^{-1}(Y-Z)}$ is smooth.

( 5 ) $\mathrm{Ch}\left(\mathscr{M}^{*}\right) \subset T_{Y}^{*} X \cup Z \underset{X}{\times} T^{*} X$.

(6) $Z^{\prime}=Y^{\prime} \cap f^{-1}(Z)$ is a normally crossing hypersurface of $Y^{\prime}$.

(7) $Z$ is stable under the complex conjugation.

( 8 ) $N=Y^{\prime} \cap M$ is a real analytic manifold and $Y^{\prime}$ is its complexification.

Set $\mathscr{M}^{\prime}=\boldsymbol{R} \Gamma_{\left[X^{\prime} \mid Z^{\prime}\right]}\left(\boldsymbol{L} f^{*} \mathscr{M}^{*}\right)^{*}$. Here $\mathscr{M}^{*}$ denote $\boldsymbol{R} \mathscr{H} o m_{\mathscr{D}_{X}}\left(\mathscr{M}^{*}\right.$, $\left.\mathscr{D}_{X}\right) \underset{\mathscr{D}_{X}}{\otimes} \Omega_{X}^{\otimes-1}[\operatorname{dim} X]$, and $\boldsymbol{L} f^{*} \mathscr{M}^{*}=\mathscr{D}_{X^{\prime} \rightarrow X} \stackrel{\bigotimes}{\mathscr{D}}_{X}^{\boldsymbol{L}} \mathscr{M}^{* *}$.

Lemma 8. 3. 1 .

(1) $\boldsymbol{R} f_{*} \boldsymbol{R} \mathscr{H o m}_{\mathscr{D}_{X}}\left(\mathscr{M}^{\prime}, \mathscr{D} b_{M^{\prime}}\right)=\boldsymbol{R} \mathscr{H o m}_{\mathscr{D}_{X}}\left(\boldsymbol{R} \Gamma_{[X \mid Z]}\left(\mathscr{M}^{*}\right)^{*}, \mathscr{D} b_{M}\right)$

(2) $\boldsymbol{R} f_{*} \boldsymbol{R} \mathscr{H o m _ { \mathscr { D } _ { X } }}\left(\mathscr{M}^{\prime}, \mathscr{B}_{M^{\prime}}\right)=\boldsymbol{R} \mathscr{H}_{0 m_{\mathscr{D}_{X}}}\left(\boldsymbol{R} \Gamma_{[X \mid Z]}\left(\mathscr{M}^{*}\right)^{*}, \mathscr{B}_{M}\right)$

Admitting this lemma for a while, we shall prove Theorem for $F=\boldsymbol{C}_{M}$.

Now, $\mathscr{M}^{\prime}$ is locally isomorphic to the direct product of

$$
\mathscr{D}_{X^{\prime}} / \sum_{j \leq l} \mathscr{D}_{X^{\prime}}\left(x_{j} \partial_{j}-\lambda_{j}\right)^{m_{j}}+\sum_{s>j>l} \mathscr{D}_{X^{\prime}} \partial_{j}+\sum_{j \geqq s} \mathscr{D}_{X^{\prime}} x_{j}[-r]
$$

for a real coordinate $\left(x_{1}, \ldots, x_{n}\right)$ of $X$. In this case, one can easily verify the theorem. Hence we obtain

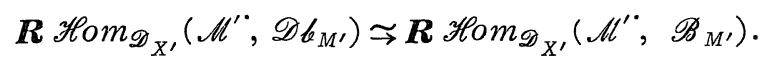

Thus by Lemma 8.3.1, we have

$$
\boldsymbol{R} \mathscr{H o m}_{\mathscr{D}_{X}}\left(\mathscr{L}^{\cdot}, \mathscr{D} b_{M}\right) \widetilde{R} \mathscr{H} o m_{\mathscr{D}_{X}}\left(\mathscr{L}^{*}, \mathscr{B}_{M}\right)
$$

for $\mathscr{L}^{*}=\boldsymbol{R} \Gamma_{[X \mid Z]}\left(\mathscr{M}^{*}\right)^{*}$. Since $\mathscr{L}^{*}$ is isomorphic to $\mathscr{M}^{*}$ on $X-Z$, there exists a triangle

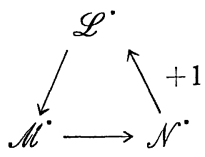

where $\operatorname{Supp} \mathscr{N}^{*} \subset Z$.

Since the theorem is true for $\mathscr{r}^{*}$ by the hypothesis of the 
induction, that is true also for $\mathscr{M}^{\circ}$.

8. 4. Proof of Lemma 8. 3.1 (1).

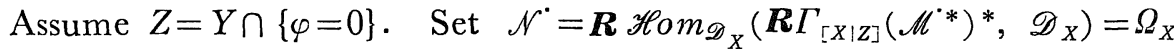

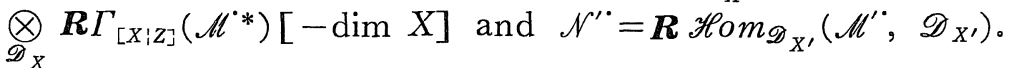

Then the right hand side of (1) is $\boldsymbol{R} f_{*}\left(\mathscr{N}^{\prime}{\underset{\mathscr{D}}{X^{\prime}}}_{L}^{L} \mathscr{D} b_{M^{\prime}}\right)$ and the left hand side is $\mathscr{N}^{\cdot} \bigotimes_{\mathscr{D}_{X}}^{L} \mathscr{D} b_{M}$.

On the other hand, we have

$$
\mathscr{N}^{*}=\mathscr{N}^{*} \otimes_{\mathscr{D}_{X}}^{L}\left(\mathscr{D}_{X}\right)_{\varphi} \quad \text { and } \quad \mathscr{N}^{\prime}=\mathscr{N}^{\cdot}{\underset{\mathscr{D}}{X}}_{X}\left(\mathscr{D}_{X^{\prime}}\right)_{\varphi^{*}}
$$

Hence we obtain by Proposition 3. 23

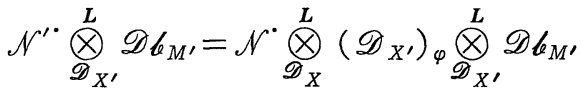

$$
\begin{aligned}
& =\mathscr{N}^{\cdot} \otimes_{\mathscr{D}_{X}} T H\left(\boldsymbol{C}_{M^{\prime}-f^{-1}(Z)}\right)
\end{aligned}
$$

and similarly

$$
\mathscr{N} \otimes_{\mathscr{D}_{X}}^{L} \mathscr{D} b_{M}=\mathscr{N}_{\mathscr{D}_{X}}^{\stackrel{L}{\bigotimes}} T H\left(\boldsymbol{C}_{M-Z}\right) .
$$

Thus, (1) follows from

$$
\boldsymbol{R} f_{*}\left(T H_{M^{\prime}}\left(\boldsymbol{C}_{M^{\prime}-\varphi^{-1}(0)}\right)\right)=T H_{M}\left(\boldsymbol{C}_{M-\varphi^{-1}(0)}\right) .
$$

Proof of Lemma 8.3.1 (2). By the argument preceding Corollary 8. 4 ,

$$
\boldsymbol{R} \mathscr{H} o m_{\mathscr{D}_{X}}\left(\mathscr{M}, \mathscr{B}_{M}\right)=\boldsymbol{R} \mathscr{H} o m_{\boldsymbol{C}_{M}}\left(\left.D R_{X}(\mathscr{M})\right|_{M}, \boldsymbol{C}_{M}\right) .
$$

Hence (2) follows immediately from

$$
D R_{X}\left(\boldsymbol{R} I_{[X \mid Z]}^{\prime}\left(\mathscr{M}^{*}\right)^{*}\right)=D R_{X}\left(\mathscr{M}^{*}\right)_{X-Z} .
$$

8. 5. Now, we shall prove Theorem 8.2 for an arbitrary $F$. We may assume that $F^{*}=\boldsymbol{C}_{Z}$ for a compact subanalytic set $Z$. We shall prove Theorem 8.2 by the induction of $\operatorname{dim} Z$. By the definition, there exists a proper real analytic map $f: N \rightarrow M$ such that $f(N)=Z$. We may assume $M$ and $N$ are oriented. Letting $Y$ a complexification of $N$ we extend $f$ to a holomorphic map $Y \rightarrow X$.

By Theorem 4. 1, we have

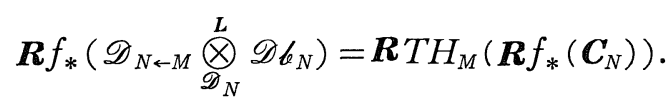


Hence

$$
\begin{aligned}
& \boldsymbol{R} \mathscr{H o m}_{\mathscr{D}_{X}}\left(\mathscr{M}, \boldsymbol{R} T H_{M}\left(\boldsymbol{R} f_{*}\left(\boldsymbol{C}_{N}\right)\right)\right. \\
& =\boldsymbol{R} \mathscr{H o m}_{\mathscr{D}_{X}}\left(\mathscr{M}^{\circ}, \mathscr{D}_{X}\right){\underset{\mathscr{D}}{X}}_{X}^{\boldsymbol{L}} \boldsymbol{R} T H_{M}\left(\boldsymbol{R} f_{*}\left(\boldsymbol{C}_{N}\right)\right) \\
& =\boldsymbol{R} \mathscr{H}_{0 m_{\mathscr{D}_{X}}}\left(\mathscr{M} ; \mathscr{D}_{X}\right){\underset{\mathscr{D}}{X}}_{X}^{\boldsymbol{L}} \boldsymbol{R} f_{*}\left(\mathscr{D}_{M \leftarrow N} \bigotimes_{\mathscr{D}_{N}}^{\boldsymbol{L}} \mathscr{D} b_{N}\right)
\end{aligned}
$$

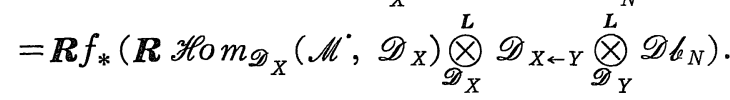

This holds also by replacing $\mathscr{D} k$ with $\mathscr{B}$. Hence Theorem 8.2 for the constant sheaf impiles

$$
\begin{aligned}
& \boldsymbol{R} \mathscr{H o}_{\mathscr{D}_{X}}\left(\mathscr{M}^{\circ}, \boldsymbol{R} T H_{M}\left(\boldsymbol{R} f_{*}\left(\boldsymbol{C}_{N}\right)\right)\right) \\
& \quad=\boldsymbol{R} \mathscr{H} o m_{\mathscr{D}_{X}}\left(\mathscr{M}^{\circ}, \boldsymbol{R} \mathscr{H} o m_{c_{M}}\left(\boldsymbol{R} f_{*}\left(\boldsymbol{C}_{N}\right), \mathscr{B}_{M}\right)\right) .
\end{aligned}
$$

Now there exists a nowhere dense closed subanalytic subset $Z^{\prime} \subset Z$ such that

(1) $\left.\quad \boldsymbol{R} f_{*}\left(\boldsymbol{C}_{N}\right)\right|_{z-z^{\prime}}$ is constant.

(2) $\left.\quad F^{*}\right|_{z-Z^{\prime}}$ is constant.

By the hypothesis of induction, the theorem is true for $F_{Z^{\prime}} \cdot \boldsymbol{R} f_{*}\left(\boldsymbol{C}_{N}\right)_{Z^{\prime}}$, and hence it is true for $\boldsymbol{R} f_{*}\left(\boldsymbol{C}_{Z}\right)_{Z-Z^{\prime}}$. Since $\boldsymbol{R} f_{*}\left(\boldsymbol{C}_{Z}\right)_{Z-Z^{\prime}}$ is constant the theorem is true for $\boldsymbol{C}_{Z-Z^{\prime}}$ and hence for $F_{Z-Z^{\prime}}$. Thus we obtain the desired result.

\section{References}

[Hl] Hironaka, H., Resolution of singularities of an algebraic variety over a field of characteristic 0, Annals of Math., 79 (1964), 109-326.

[H2] Subanalytic sets, Number theory, algebraic geometry and commutative algebra, in honor of Y. Akizuki, Kinokuniya, Tokyo (1973), 453-493.

[H3] - Introduction aux ensembles sous-analytiques, Astérisque 7 et 8, Société math. de France (1973), 13-20, rédigé par A. Hirschowitz and P. LeBarz.

[K] Kashiwara, M., Faisceaux constructibles et systèmes holonomes d'équations aux dérivées partielles linéaires à points singuliers réguliers, Sém. Goulaouic-Schwartz, 1979-80, exposé 19.

[K1] On the maximally overdetermined system of linear differential equations, I, Publ. RIMS, Kyoto Univ., 10 (1975) 563-579.

[K2] - B-functions and holonomic systems, Invent. Math., 38 (1976), 33-53.

[KK] Kashiwara, M. and Kawai, T., On holonomic systems of microdifferential equations III-system with regular singularities, Publ. RIMS, Kyoto Univ., 17 (1981), 813-979.

[L] Lojasiewicz, S., Sur le problème de la division, Studia Math., 8 (1959), 87-136.

[Ma] Martineau, A., Distributions et valeurs au bord des fonctions holomorphes, Oeuvre de André Martineau, Paris, GNRS, (1977), 439-582.

[Me] Mebkout, Z., Sur le problème de Riemann-Hilbert, Lecture Notes in Physics, 126, 
Berlin-Heidelberg-New York, Springer (1980), 99-110.

[S] Schwartz, L., Théorie des distributions, Paris, Hermann (1950).

[SKK] Sato, M., Kawai, T. and Kashiwara, M., Microfunctions and pseudo-differential equations, Lecture Notes in Math., 649, Berlin-Heidelberg-New York, Springer (1978), 228-289.

[V] Verdier, J. L., Dualité dans la cohomologie des espaces localement compacts, Sém. Bourbaki, exposé 300 (1965).

\section{Added in proof:}

The proof of Theorem 2.8 is not complete because we assumed $\operatorname{dim} F_{x}<\infty$ for an $\boldsymbol{R}$-constructible sheaf $F$ but not for an $S$-constructible sheaf $F$. This difficulty can be overcome by one of the following methods. The first method relies on the fact that Theorem 2.8 is proven if we replace " $\boldsymbol{R}$-constructible" with "weakly $\boldsymbol{R}$-constructible" and that the functor $T H$ is a well-defined exact functor on the category of weakly $\boldsymbol{R}$-constructible sheaves. The second method is to prove Theorem 2.8 in the original form by using the following lemma which can be easily shown.

Lemma. If $F^{*}$ is a bounded complex of $S$-constructible sheaves on $|\mathscr{S}|$ such that $\operatorname{dim} \mathscr{H}^{j}\left(F^{*}\right)_{x}$ $<\infty$, then $F^{*}$ is quasi-isomorphic to a bounded complex $F^{\prime}$ of $S$-constructible sheaves such that $\operatorname{dim} F_{x}^{\prime j}<\infty$. 
